\title{
A scoping review of severe maternal morbidity: describing risk factors and methodological approaches to inform population-based surveillance
}

Lisa M. Korst ${ }^{1 *}$ (D) Kimberly D. Gregory ${ }^{2,3,4}$, Lisa A. Nicholas ${ }^{3}$, Samia Saeb², David J. Reynen $^{5}$, Jennifer L. Troyan ${ }^{5}$, Naomi Greene ${ }^{2}$ and Moshe Fridman ${ }^{6}$

\begin{abstract}
Background: Current interest in using severe maternal morbidity (SMM) as a quality indicator for maternal healthcare will require the development of a standardized method for estimating hospital or regional SMM rates that includes adjustment and/or stratification for risk factors.

Objective: To perform a scoping review to identify methodological considerations and potential covariates for risk adjustment for delivery-associated SMM.

Search methods: Following the guidelines for Preferred Reporting Items for Systematic Reviews and Meta-analyses Extension for Scoping Reviews, systematic searches were conducted with the entire PubMed and EMBASE electronic databases to identify publications using the key term "severe maternal morbidity."

Selection criteria: Included studies required population-based cohort data and testing or adjustment of risk factors for SMM occurring during the delivery admission. Descriptive studies and those using surveillance-based data collection methods were excluded.

Data collection and analysis: Information was extracted into a pre-defined database. Study design and eligibility, overall quality and results, SMM definitions, and patient-, hospital-, and community-level risk factors and their definitions were assessed.

Main results: Eligibility criteria were met by 81 studies. Methodological approaches were heterogeneous and study results could not be combined quantitatively because of wide variability in data sources, study designs, eligibility criteria, definitions of SMM, and risk-factor selection and definitions. Of the 180 potential risk factors identified, 41 were categorized as pre-existing conditions (e.g., chronic hypertension), 22 as obstetrical conditions (e.g., multiple gestation), 22 as intrapartum conditions (e.g., delivery route), 15 as non-clinical variables (e.g., insurance type), 58 as hospital-level variables (e.g., delivery volume), and 22 as community-level variables (e.g., neighborhood poverty).

(Continued on next page)
\end{abstract}

\footnotetext{
* Correspondence: korst@sbcglobal.net

${ }^{1}$ Childbirth Research Associates, LLC, North Hollywood, CA, USA

Full list of author information is available at the end of the article
}

(c) The Author(s). 2020 Open Access This article is licensed under a Creative Commons Attribution 4.0 International License, which permits use, sharing, adaptation, distribution and reproduction in any medium or format, as long as you give appropriate credit to the original author(s) and the source, provide a link to the Creative Commons licence, and indicate if changes were made. The images or other third party material in this article are included in the article's Creative Commons licence, unless indicated otherwise in a credit line to the material. If material is not included in the article's Creative Commons licence and your intended use is not permitted by statutory regulation or exceeds the permitted use, you will need to obtain permission directly from the copyright holder. To view a copy of this licence, visit http://creativecommons.org/licenses/by/4.0/. The Creative Commons Public Domain Dedication waiver (http://creativecommons.org/publicdomain/zero/1.0/) applies to the data made available in this article, unless otherwise stated in a credit line to the data. 
(Continued from previous page)

Conclusions: The development of a risk adjustment strategy that will allow for SMM comparisons across hospitals or regions will require harmonization regarding: a) the standardization of the SMM definition; b) the data sources and population used; and c) the selection and definition of risk factors of interest.

Keywords: Severe maternal morbidity, Maternal care, Obstetrics, Blood transfusion, Disparities, Quality indicators

\section{Introduction}

The tracking of severe maternal morbidity (SMM) has continued to evolve since it was first initiated by the World Health Organization (WHO) in 2004 as an alternative to maternal mortality surveillance for identifying failures and priorities in maternal health care [1]. By 2009, the WHO adopted a definition for a maternal near-miss (i.e., "a woman who nearly died but survived a complication that occurred during pregnancy, childbirth or within 42 days of termination of pregnancy") and presented a list of identification criteria [2]. Using this approach, cases are first identified as having "potentially life-threatening conditions" associated with organ system dysfunction or failure. Surveillance relies on medical record review to document clinical, laboratory-based, or management-based SMM criteria. Case identification can occur retrospectively by identifying those who met criteria or prospectively by using a list of potentially lifethreatening conditions.

A population-based approach to tracking SMM began in parallel to the WHO approach in 2005, when Wen et al. proposed a definition of SMM using population-based Canadian administrative data [3], which, although less specific compared to medical record data, was more feasible for routine monitoring. In 2008, Callaghan et al. at the United States (US) Centers for Disease Control and Prevention (CDC) published a definition using 15 conditions [4]. The CDC definition was expanded to 25 conditions in 2012 [5], and in 2015 when International Classification of Diseases, Clinical Modification, Version 9 (ICD-9) coding was upgraded to ICD-10, the SMM definition was reduced and consolidated to 21 and then to 18 conditions [6]. Roberts et al. in Australia contributed substantially to these efforts [7], and this work was further adapted in Canada by Joseph et al. [8]. Canadian and Australian SMM definitions were developed in ICD-10.

In the US, in addition to the CDC calculations of national, population-based trends for SMM using administrative data, facility-based SMM case audit (here referred to as "facility-based surveillance") has been encouraged by both the CDC and the American College of Obstetricians and Gynecologists $[9,10]$. In a recent review, Kuklina and Goodman promoted these complementary approaches, asserting that while case audits can go into depth to identify the causes of SMM and suggest avenues for prevention, population-based administrative data can be used not only to examine trends, but also to compare "hospitals, cities, or states" and to develop priorities for research and practice [11]. With funding from the Centers for Medicare and Medicaid services, the National Quality Forum, which provides standards for healthcare quality measurement in the US, has begun to explore the use of maternal morbidity and mortality measures to improve outcomes [12].

To make comparisons (e.g., by region or hospital) interpretable and amenable to policy directives and interventions, it will be necessary to develop a standardized method for adjusting for the most relevant risk factors. To address the research question of how an SMM measure might be adjusted for such comparisons, the objective of this scoping review was to describe what is currently known regarding the risk factors and methodological approaches for studying SMM using routinely collected population-based data.

\section{Materials and methods}

\section{Data sources and search strategy}

We performed a scoping literature review for predictors of delivery-admission SMM, using the definition provided by Anderson et al. [13]: "Scoping studies are concerned with contextualizing knowledge in terms of identifying the current state of understanding; identifying the sorts of things we know and do not know; and then setting this within policy and practice contexts." This review conformed to guidelines for Preferred Reporting Items for Systematic Reviews and Metaanalyses Extension for Scoping Reviews (PRISMA-ScR) [14]. The PRISMA-ScR checklist is available in Additional file 1. Using the search term "severe maternal morbidity," the search was conducted in PubMed and Embase, (both initiated in the late 1940's), and included the entire databases through June 9, 2019. Since SMM is a composite measure that may include as few as one (e.g., intensive care unit admission) or more than 25 indicators, we used only this term (SMM) to narrow our search and retrieve articles using SMM definitions that were most relevant to population-based SMM tracking. 


\section{Inclusion and exclusion criteria}

The inclusion criteria were that each study examined SMM as an outcome occurring during the delivery admission and that SMM was presented with risk factor adjustment or stratification. The exclusion criteria were the following: 1) case reports; 2) reviews, letters, or editorials; 3) errata; 4) methodological studies; 5) protocol only studies; 6) descriptive only studies; 7) quality improvement studies; 8) limited outcome studies (i.e., those that did not include a comprehensive SMM composite); 9) studies using SMM as a risk factor/predictor for further complications; 10) qualitative studies; 11) readmission SMM studies; 12) studies focused on SMM due to gynecological conditions (e.g., ectopic pregnancy or spontaneous abortion); 13) studies that relied predominantly on risk factors that were not routinely available in administrative data (e.g., clinical data, laboratory test results); 14) studies that relied on surveillance-based data collection methods (e.g., WHO-based methods); and 15) studies that were not in English. Citations identified through the searches were assessed by one reviewer and verified by another based on title and abstract using these pre-defined criteria. Duplicates were removed. Full text publications of potentially relevant citations were then examined by the same reviewers to assure that eligibility criteria were met.

\section{Data extraction}

Reviewer disagreements about study selection in the full text review and extraction phases were resolved by jointly re-examining studies and reaching mutual agreement. Information regarding each study was then extracted into a pre-defined database. Variables extracted included: study design and eligibility criteria; datasets used; SMM definition used; and risk factors used, including all patient-, hospital-, and community-level risk factors as reported.

\section{Quality assessment}

Risk of bias assessment is not a mandatory part of this review; however, for informative purposes, risk of bias was assessed using a version of the Newcastle-Ottawa Scale (NOS) [15] that was modified to evaluate observational cross-sectional studies relevant to the research question (Additional file 2). Risk of bias was assessed for a single outcome (SMM) within a study. Two reviewers assessed the articles for NOS criteria. Any discrepancy in scoring was resolved by joint re-examination to arrive at consensus. NOS scores were subdivided into those indicating high- (7-10), moderate- (5-6), and low-quality (1-4) of the publication with respect to the research question. Quality scores were used as part of the general assessment of the literature and did not affect the synthesis of results.

\section{Synthesis of results}

Risk factors were categorized into patient-, hospital-, and community-level groups. The patient-level risk factors were classified as pre-existing conditions (e.g., medical or behavioral risk factors), obstetrical conditions (e.g., multiple gestation), and non-clinical conditions (e.g., insurance type). The obstetrical conditions were further divided into those known to exist in the antepartum period (e.g., prior cesarean birth) versus those occurring in the intrapartum or postpartum periods of the delivery admission (e.g., dystocia, delivery mode).

The heterogeneity of the studies included in this review precluded any quantitative synthesis of the effect sizes (odds ratios or relative risks) of the identified risk factors. However, we present a comprehensive list of the risk factors for SMM that were reported in these publications and summarize: 1) the number of studies that used each risk factor; 2) the number of studies for which the effect size for a risk factor was reported to be statistically significant; and 3) the number of studies that did not report an effect size for the risk factor but did one of the following: a) included it in a risk adjustment model, b) stratified analyses by the risk factor, or c) excluded subsets of the population based on the risk factor. This information is presented with the understanding that it was not meaningful to compare or aggregate effect sizes across studies because of the wide heterogeneity of the study designs and covariates in the models.

The synthesis of results was organized by placing the 81 studies in the following categories defined by study approach: 1) Testing of multiple conditions for association with SMM without invoking a specific hypothesis; 2) Hypothesis testing of specific patient-level risk factors; 3) Hypothesis testing of hospital-level risk factors; and 4) Risk-adjusted SMM rates and trends. For purposes of this review, both maternal age and race/ethnicity were treated as pre-existing clinical risk factors.

\section{Results}

\section{Study selection and characteristics}

Results of the literature search are described in Fig. 1. A total of 1489 publications were identified by the original searches, and, of 861 unique titles, 81 (9.4\%) met all inclusion and exclusion criteria after thorough review of the full text $[3,8,16-94]$. Table 1 summarizes selected study characteristics and Table 2 presents the risk factors used in the models. Most of the studies $(n=71$, 87.7\%) relied on hospital claims data, medical records, or discharge/birth certificate data from US hospitals. Although some studies sought to examine and identify general clinical risk factors as defined above $(n=16$, $19.8 \%$ ), most ( $n=57,70.4 \%$ ) hypothesized an association 


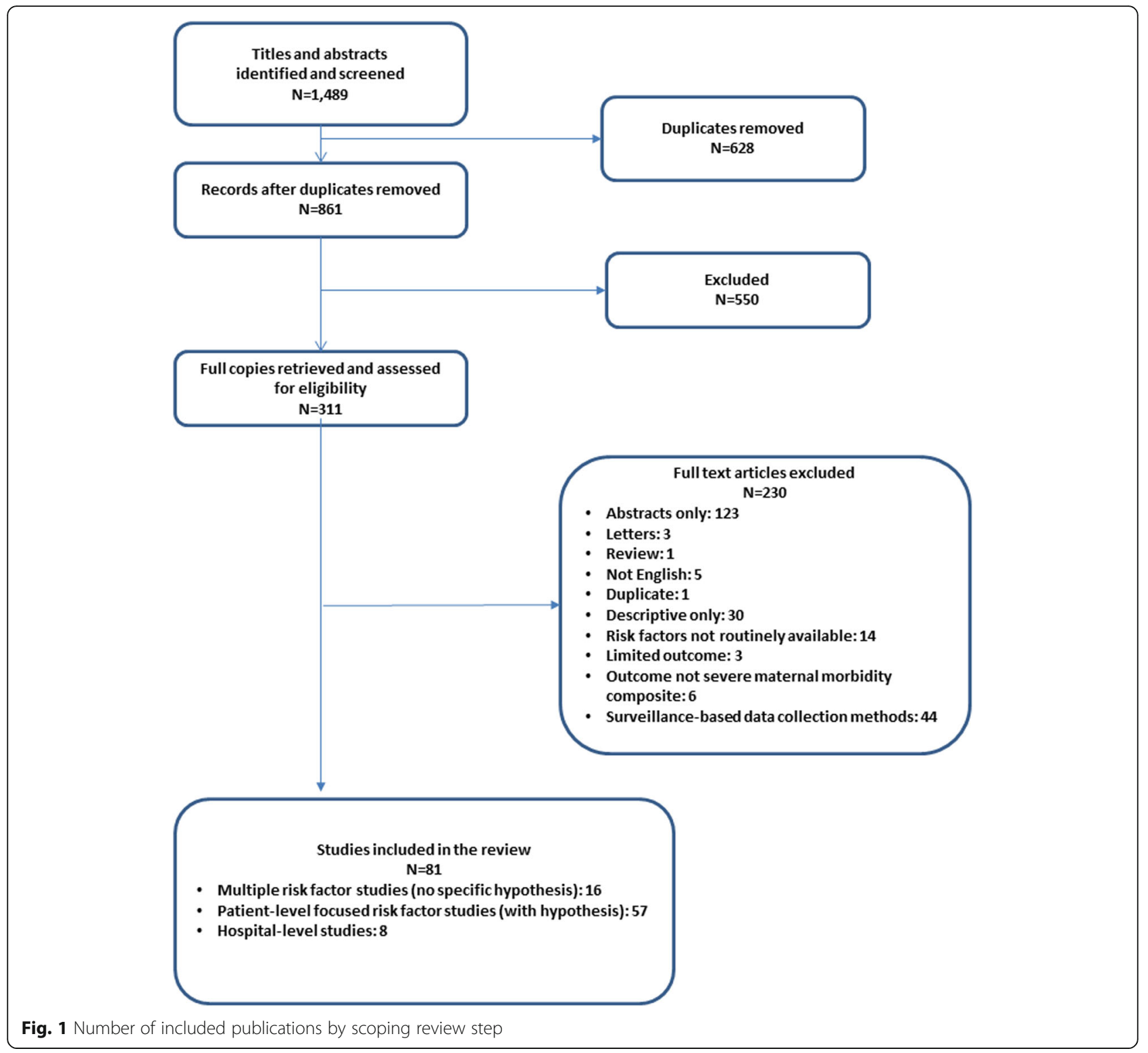

of SMM with a specific risk factor, such as maternal age or race/ethnicity, or other pre-existing clinical condition; eight studies (9.9\%) examined hospital-level risk factors, such as delivery volume. Over one-quarter of the studies ( $n=22,27.2 \%)$ limited the study population by patient characteristics (e.g., women at low risk) or hospital type (e.g., community hospitals).

\section{Risk of bias of included studies}

Quality scoring is presented in Table 3. Of 10 potential points per study, the median score was 6 (range 3-10). There were 37 high-quality, 39 moderate-quality, and 5 low-quality studies.

\section{Synthesis of results by study approach}

\section{Testing of multiple conditions for association with SMM}

The 16 publications in this category are described in Table 2. Four of these publications attempted to describe the accuracy of the models using various statistical techniques [22, 43, 44, 83]. All studies used maternal age and 11 used race/ethnicity. In several studies, maternal age $[3,27,33,47,69,81,82]$ and parity $[3,42,53,82]$ appeared to have a $\mathrm{U}$ - or J-shaped relationship with $\mathrm{SMM}$, requiring categorization into three or more groups or appropriate selection of the functional form (e.g., polynomial or logistic) for the association of these covariates with SMM. Two studies used no pre-existing risk factors [3, 90] and one used no obstetrical risk 
Table 1 Summary of included studies with reference numbers. $N=81$ [3, 8, 16-94]

\section{Study Characteristic}

Number and data source of included studies

US $^{\mathrm{a}}$ data $(n=50)$

National Inpatient Sample

National Readmission Database: 21 states

$\mathrm{CMS}^{\mathrm{b}}$ Medicaid (MAX) data

US claims data

US perinatal data networks

Hospital discharge data: 7 states

Hospital discharge data: Illinois

Hospital discharge data: Maryland

Hospital discharge \pm birth certificate data: New York State

Hospital discharge + birth certificate data: 3 states

Hospital discharge + birth certificate data: lowa

Hospital discharge + birth certificate data: New York City

Hospital discharge, birth, \pm death certificate data: California

Hospital discharge, birth, + death certificate data: Georgia

Hospital discharge, birth, \pm death certificate data: Washington

Hospital discharge + medical record data: 16 California hospitals

Hospital discharge + medical record data: Massachusetts

Hospital discharge + SART ${ }^{\mathrm{c}}+$ vital statistics data: Massachusetts

Birth certificate data: Ohio

Birth certificate and SART data: 8 states

Medical record data single hospital: California

Medical record data single hospital: New York

Medical record data single hospital: Missouri

Medical record data single hospital: Tennessee

Non-US data $(n=31)$

Multiple countries including US

Multiple countries not including US

Japan

Korea

Sweden

Finland

France

Canada: Ontario

Canada: British Columbia

Canada: multiple provinces

Australia: New South Wales

Australia: Victoria
$17,18,26,39,45,48,58,72$

Reference Number

32

22

27,70

28,43

33

84

81

$19,44,53$

29

38

$46,47,49,50,78$

$36,40,52,55-57,68,69,76$

91

$42,62-64$

51

21

23

71

67

93

73

83

41

60

90

16

75

92

77

$25,54,88$

$61,85,86$

$3,8,65,66,74,79,94$

$20,24,30,31,37,82,87$

59

$16,21,34,42,54,88$
$34,35,80,89$

\section{Study design and population}

Study design nested case-control $(n=6)$

Yes 
Table 1 Summary of included studies with reference numbers. $N=81[3,8,16-94]$ (Continued)

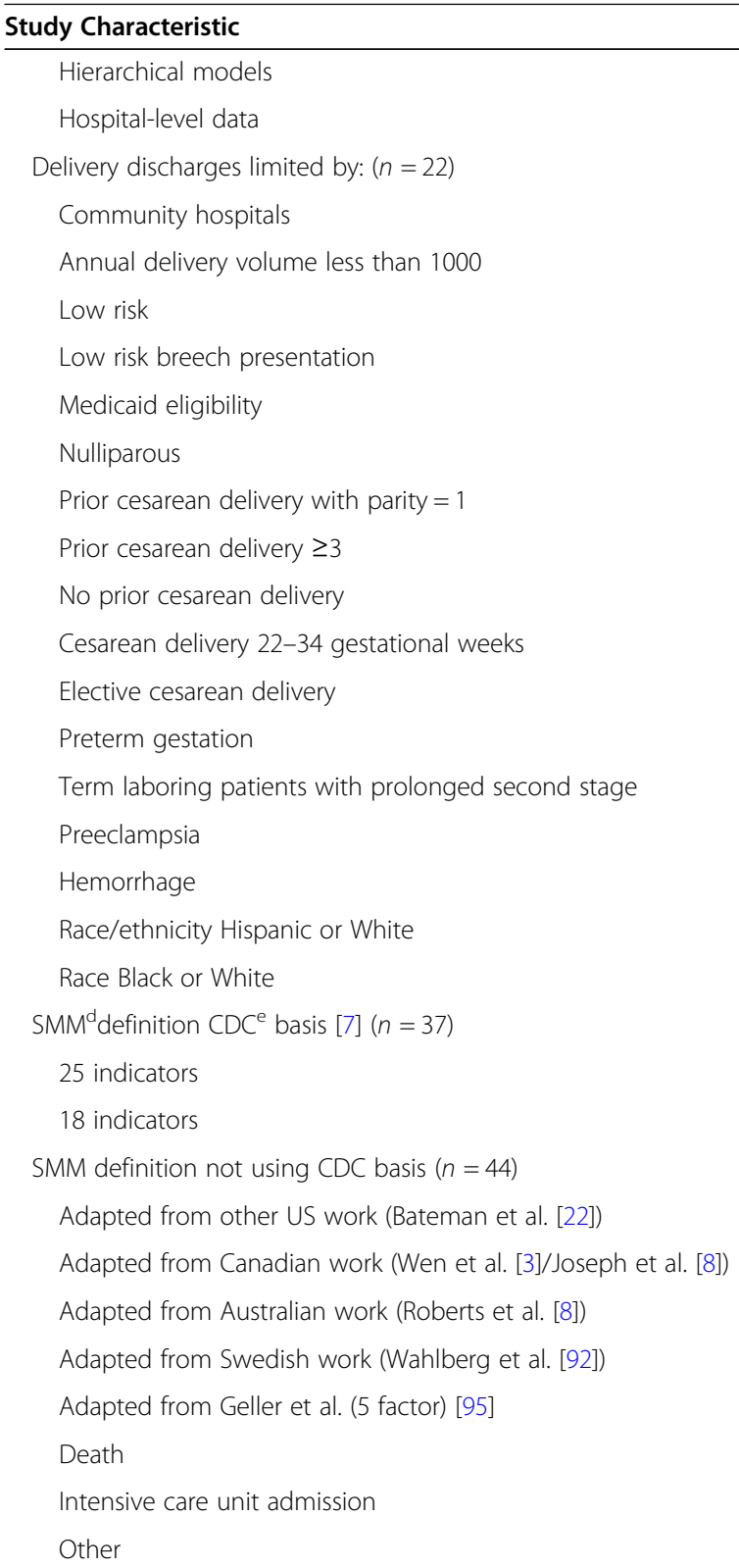

\section{Key risk factors studied}

General risk factors (no hypotheses) $(n=16)$

$$
\text { Yes }
$$

Patient-level risk factors with specific hypotheses $(n=57)$

Race/ethnicity

$\mathrm{BMI}^{\mathrm{f}}$ or gestational weight gain

\section{Reference Number}

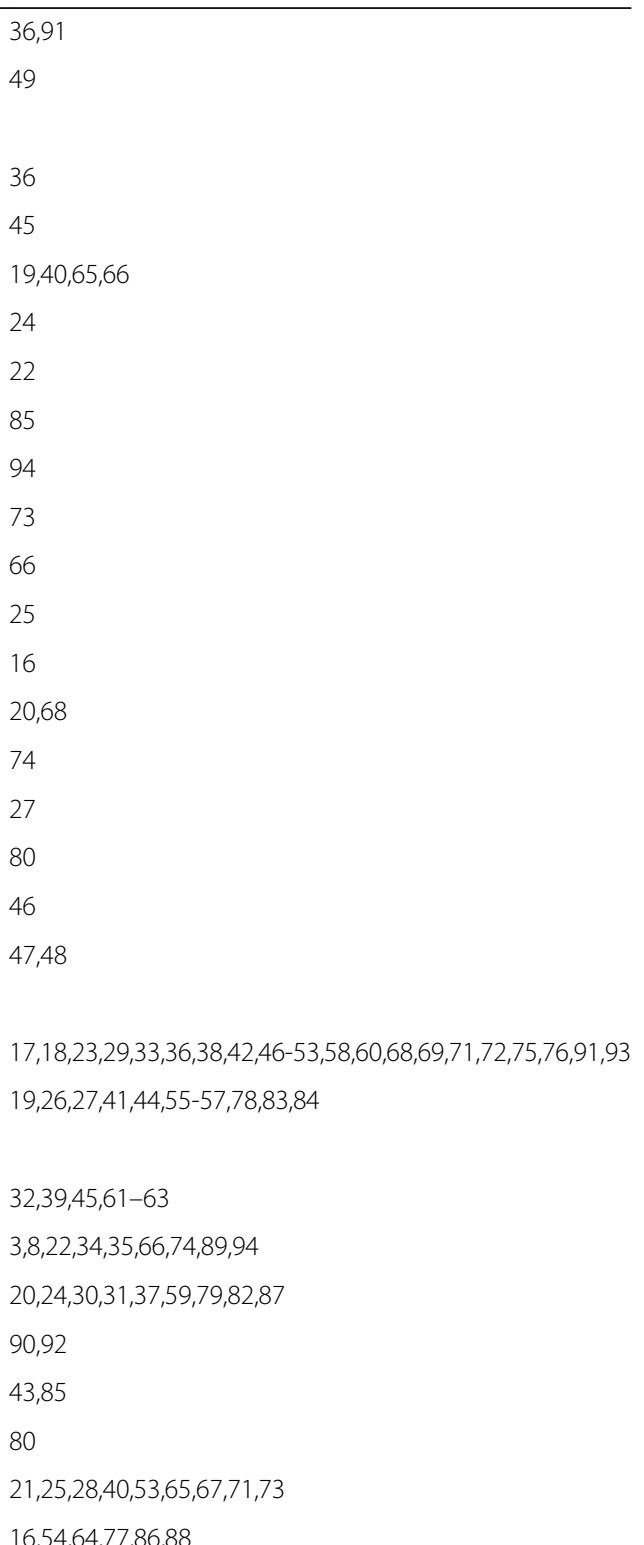

$16,54,64,77,86,88$

$26,33,38,44,46,47,49,50,55-57,60,78,91,94$

$43,51,54,85,88,93$

$22,32,37,39,45,77,80,86,90,92$

$3,8,22,38,42-45,52,53,59,60,80,81,83,90$

$18,26,33,46-48,50,57,58,72$

$35,55,62,71,73,78,86,88$ 
Table 1 Summary of included studies with reference numbers. $N=81[3,8,16-94]$ (Continued)

\begin{tabular}{|c|c|}
\hline Study Characteristic & Reference Number \\
\hline Maternal age & $63,76,85$ \\
\hline Preterm birth & $25,51,68$ \\
\hline Infertility/IVFg/ART ${ }^{\mathrm{h}}$ & $23,34,54,67,70,93$ \\
\hline Preeclampsia & 64 \\
\hline Hemorrhage & 37 \\
\hline Obstructive sleep apnea & 28 \\
\hline Congenital heart disease & 79 \\
\hline Inflammatory bowel disease & 87 \\
\hline Autoimmunity & 30 \\
\hline Idiopathic arthritis & 31 \\
\hline Rural vs. urban & 61 \\
\hline Maternal birthplace or immigration country & 89,92 \\
\hline Amphetamine/opioid use & 17 \\
\hline Route of delivery & $20,21,24,41,56,66,74,77,84,94$ \\
\hline Induction of labor & $40,65,82$ \\
\hline Anesthesia for cesarean delivery & 16 \\
\hline Off-hours delivery & 69,75 \\
\hline \multicolumn{2}{|c|}{ Hospital-level risk factors with specific hypotheses $(n=8)$} \\
\hline Delivery volume & $27,29,39$ \\
\hline Level of care & 32,91 \\
\hline Percent midwives delivering & 19 \\
\hline Presence of laborist & 36 \\
\hline Hospital quality indicators & 49 \\
\hline
\end{tabular}

a US United States; ${ }^{\mathrm{b}}$ CMS Centers for Medicare and Medicaid Services; ${ }^{\mathrm{c}}$ Society for Assisted Reproductive Technology; ${ }^{\mathrm{d}} \mathrm{SMM}$ severe maternal morbidity; ${ }^{\mathrm{e}} \mathrm{CDC}$ Centers for Disease Control and Prevention; ${ }^{\mathrm{f}} B M I$ body mass index; ${ }^{\mathrm{g}} / V F$ in vitro fertilization; ${ }^{\mathrm{h}} A R T$ assisted reproductive technology

factors [60]. Of the 15 studies that did use obstetrical risk factors, four included intrapartum risk factors $[3,38$, $44,53]$.

\section{Hypothesis testing of specific risk factors for SMM}

There were 57 studies in this category, and the key risk factors used in modelling are listed in Table 2. As with the studies that tested multiple conditions, maternal age and race/ethnicity were common covariates, as was body mass index (BMI). Where BMI was treated as an independent risk factor, it appeared to have a U-shape. Patients who were underweight and those who were obese had increased risk [42, 53, 55, 62, 63].

There were 10 publications that focused specifically on race $[18,26,33,46-48,50,57,58,72]$. SMM rates of Black women have been found to be higher than those of White women, even among those with no comorbidities. In a study by Admon et al., among women with no physical or behavioral health conditions, the SMM rate of non-Hispanic Black women was nearly twice that of non-Hispanic White women [18]. Among women with two or more chronic health conditions, non-Hispanic
Black women again had an SMM rate that was nearly twice the rate of non-Hispanic White women. Viewed another way, over time, Metcalfe et al. examined trends of SMM rates by race/ethnicity and found that adjustment for race did not change the SMM trends for 5-year periods between 1993 and 2012, over and above adjustment for comorbidity [72]. Similarly, Leonard et al., in a California study [26], and Booker et al., in a study of older women [57], examined SMM rates over time and found that all racial groups experienced rising SMM; SMM was strongly affected by the presence of comorbidities; and the SMM increases for Black and White women were proportionate. Furthermore, Howland et al. demonstrated that Black-White disparities persisted in the highest income and educational groups [50]. Taken together, these studies suggest that there is a baseline difference in SMM between Black and White women that has not been explained.

Several studies [23, 34, 54, 67, 70, 93] tested whether infertile women were at increased risk of SMM. All found an increased risk for SMM among those receiving infertility treatments, cautioning that this increased risk 
Table 2 Risk factors tested for association with severe maternal morbidity

\begin{tabular}{|c|c|c|c|c|}
\hline Potential covariates for use in risk adjustment & $\begin{array}{l}\text { Number of studies } \\
\text { that used the } \\
\text { variable in risk } \\
\text { adjustment }\end{array}$ & $\begin{array}{l}\text { Number of studies } \\
\text { with a statistically } \\
\text { significant result }\end{array}$ & $\begin{array}{l}\text { Number of } \\
\text { studies with a } \\
\text { non-significant } \\
\text { result }\end{array}$ & $\begin{array}{l}\text { Number of studies } \\
\text { where statistical } \\
\text { significance was not } \\
\text { reported }\end{array}$ \\
\hline \multicolumn{5}{|l|}{ Pre-existing clinical } \\
\hline $\begin{array}{l}\text { Heart disease: including } \mathrm{CHF}^{\mathrm{a}, \mathrm{b}} \mathrm{CHD}_{1}^{\mathrm{a}, \mathrm{c}} \text { pulmonary } \\
\text { hypertension, }{ }^{\mathrm{a}} \text { ischemic heart disease, }{ }^{\mathrm{a}} \text { valvular } \\
\text { heart disease, }{ }^{\mathrm{a}} \text { conduction disorders }\end{array}$ & $43(53.1 \%)$ & 10 & 0 & 33 \\
\hline Sickle cell disease ${ }^{a}$ & $11(13.6 \%)$ & 1 & 0 & 10 \\
\hline $\begin{array}{l}\text { Collagen vascular disease: including } \mathrm{SLE}^{\mathrm{a}, \mathrm{d}} \text { and } \\
\text { rare autoimmune, rheumatoid arthritis and other } \\
\text { collagen vascular }\end{array}$ & $27(33.3 \%)$ & 5 & 0 & 22 \\
\hline$H I V^{a}$ & $21(25.9 \%)$ & 3 & 0 & 18 \\
\hline Chronic renal disease $^{a}$ & $37(45.7 \%)$ & 7 & 0 & 30 \\
\hline Chronic hypertension $^{a}$ & $68(84.0 \%)$ & 20 & 0 & 48 \\
\hline Chronic diabetes $^{\mathrm{a}}$ & $67(82.7 \%)$ & 15 & 1 & 51 \\
\hline Chronic lung disease, including asthma ${ }^{a}$ & $31(38.3 \%)$ & 5 & 1 & 25 \\
\hline Thyroid disease, hypothyroidism & $8(9.9 \%)$ & 0 & 0 & 8 \\
\hline $\begin{array}{l}\text { Maternal soft tissue condition: includes other } \\
\text { uterine surgery, fibroids, cervical conditions }\end{array}$ & $5(6.2 \%)$ & 0 & 0 & 5 \\
\hline Pelvis abnormal & $1(1.2 \%)$ & 0 & 0 & 1 \\
\hline $\begin{array}{l}\text { Gynecological conditions: e.g., endometriosis, } \\
\text { PCOS, peritoneal adhesions }\end{array}$ & $3(3.7 \%)$ & 0 & 0 & 3 \\
\hline Skin and subcutaneous tissues & $1(1.2 \%)$ & 0 & 0 & 1 \\
\hline $\begin{array}{l}\text { Hospitalization in prior } 5 \text { years or during } \\
\text { pregnancy }\end{array}$ & $4(4.9 \%)$ & 1 & 0 & 3 \\
\hline $\begin{array}{l}\text { Drug abuse: }{ }^{a} \text { including amphetamine, opioid, } \\
\text { other; one vs multiple substance, cocaine, } \\
\text { combination with alcohol or smoking or mental } \\
\text { health conditions }\end{array}$ & $27(33.3 \%)$ & 4 & 1 & 22 \\
\hline Alcohol abuse $e^{a}$ & $16(19.8 \%)$ & 1 & 1 & 14 \\
\hline Smoking & $34(42.0 \%)$ & 6 & 7 & 21 \\
\hline Mental health, including depression & $16(19.8 \%)$ & 4 & 0 & 12 \\
\hline Obesity class, BMI, weight gain during pregnancy & $40(49.4 \%)$ & 15 & 5 & 20 \\
\hline Height & $2(2.5 \%)$ & 0 & 0 & 2 \\
\hline Weight gain & $3(3.7 \%)$ & 2 & 1 & 0 \\
\hline Liver disorders, including failure, hepatitis B or C & $11(13.6 \%)$ & 2 & 0 & 9 \\
\hline Digestive diseases, including IBD ${ }^{g}$ & $9(11.1 \%)$ & 1 & 2 & 6 \\
\hline $\begin{array}{l}\text { Seizures and other } \mathrm{CNS}^{\mathrm{h}} \text { conditions, e.g., stroke, } \\
\text { MS }^{\mathrm{i}}\end{array}$ & $12(14.8 \%)$ & 2 & 0 & 10 \\
\hline $\begin{array}{l}\text { Blood diseases: including thrombocytopenia, } \\
\text { coagulopathy, or anaemia }\end{array}$ & $16(19.8 \%)$ & 3 & 0 & 13 \\
\hline Fluid electrolyte disorders & $2(2.5 \%)$ & 0 & 0 & 2 \\
\hline Paralysis & $2(2.5 \%)$ & 0 & 0 & 2 \\
\hline Peripheral vascular disorders & $4(4.9 \%)$ & 0 & 0 & 4 \\
\hline Weight loss & $2(2.5 \%)$ & 0 & 0 & 2 \\
\hline Musculoskeletal conditions & $5(6.2 \%)$ & 0 & 2 & 3 \\
\hline Malignancy & $4(4.9 \%)$ & 0 & 0 & 4 \\
\hline History of organ transplant & $1(1.2 \%)$ & 0 & 0 & 1 \\
\hline High risk summary measure & $28(34.6 \%)$ & 14 & 0 & 14 \\
\hline
\end{tabular}


Table 2 Risk factors tested for association with severe maternal morbidity (Continued)

\begin{tabular}{|c|c|c|c|c|}
\hline Potential covariates for use in risk adjustment & $\begin{array}{l}\text { Number of studies } \\
\text { that used the } \\
\text { variable in risk } \\
\text { adjustment }\end{array}$ & $\begin{array}{l}\text { Number of studies } \\
\text { with a statistically } \\
\text { significant result }\end{array}$ & $\begin{array}{l}\text { Number of } \\
\text { studies with a } \\
\text { non-significant } \\
\text { result }\end{array}$ & $\begin{array}{l}\text { Number of studies } \\
\text { where statistical } \\
\text { significance was not } \\
\text { reported }\end{array}$ \\
\hline $\mathrm{VTE}^{\mathrm{j}}$ anticoagulant use (now or in past) & $4(4.9 \%)$ & 1 & 0 & 3 \\
\hline Hyperlipidemia & $1(1.2 \%)$ & 0 & 0 & 1 \\
\hline Disorders of the adrenal gland & $1(1.2 \%)$ & 0 & 0 & 1 \\
\hline Obstructive sleep apnea & $1(1.2 \%)$ & 1 & 0 & 0 \\
\hline Genital herpes & $4(4.9 \%)$ & 0 & 0 & 4 \\
\hline Cystic fibrosis & $2(2.5 \%)$ & 0 & 1 & 1 \\
\hline Maternal age $\mathrm{a}^{\mathrm{a}}$ & 79 (97.5\%) & 31 & 2 & 46 \\
\hline Maternal race/ethnicity & $44(54.3 \%)$ & 24 & 0 & 20 \\
\hline \multicolumn{5}{|l|}{$N=41$ pre-existing clinical covariates } \\
\hline \multicolumn{5}{|l|}{ Obstetrical Antepartum } \\
\hline $\begin{array}{l}\text { Parity (nulliparous vs grand multipara) and } \\
\text { combinations with prior cesarean birth }\end{array}$ & $48(59.3 \%)$ & 16 & 0 & 32 \\
\hline $\begin{array}{l}\text { Prior cesarean delivery, }{ }^{a} \text { number of prior } \\
\text { cesareans }\end{array}$ & $48(59.3 \%)$ & 13 & 2 & 33 \\
\hline Prior preterm birth & $4(4.9 \%)$ & 0 & 0 & 4 \\
\hline Multiple gestation ${ }^{\mathrm{a}}$ & $69(85.2 \%)$ & 15 & 2 & 52 \\
\hline $\begin{array}{l}\text { Preeclampsia: including severe, mild, } \\
\text { gestational, eclampsia }\end{array}$ & $47(58.0 \%)$ & 13 & 1 & 33 \\
\hline Placental conditions & $25(30.9 \%)$ & 6 & 0 & 26 \\
\hline Gestational diabetes & $29(35.8 \%)$ & 4 & 5 & 20 \\
\hline $\begin{array}{l}\text { Assisted conception: invasive vs. non-invasive, } \\
\text { IUI, ovulation induction, IVF, ICSI,' diagnosed in- } \\
\text { fertility, infertility treatment }\end{array}$ & $13(16.0 \%)$ & 7 & 0 & 6 \\
\hline Neonatal congenital anomalies or cancer & $14(17.3 \%)$ & 0 & 0 & 14 \\
\hline Fetal presentation & $14(17.3 \%)$ & 2 & 2 & 10 \\
\hline$L G A^{m}$ or $S G A^{n}$ fetus & $14(17.3 \%)$ & 3 & 1 & 10 \\
\hline Oligohydramnios/polyhydramnios & $6(7.4 \%)$ & 1 & 0 & 5 \\
\hline Male fetus & $5(6.2 \%)$ & 0 & 0 & 5 \\
\hline $\begin{array}{l}\text { First trimester prenatal care, adequate prenatal } \\
\text { care }\end{array}$ & $17(21.0 \%)$ & 8 & 0 & 9 \\
\hline Provider type (at PNC, ${ }^{\circ}$ delivery) & $4(4.9 \%)$ & 1 & 0 & 2 \\
\hline Isoimmunization & $4(4.9 \%)$ & 0 & 0 & 4 \\
\hline $\begin{array}{l}\text { Number of previous livebirths, number of } \\
\text { previous miscarriages/previous miscarriage, } \\
\text { ectopic, termination }\end{array}$ & $4(4.9 \%)$ & 1 & 0 & 3 \\
\hline Prior $\mathrm{D} \& \mathrm{C}^{\mathrm{P}}$ & $1(1.2 \%)$ & 0 & 0 & 1 \\
\hline History of hemorrhage previous pregnancy & $2(2.5 \%)$ & 2 & 0 & 0 \\
\hline $\begin{array}{l}\text { History of hypertensive disorder in a previous } \\
\text { pregnancy }\end{array}$ & $1(1.2 \%)$ & 1 & 0 & 0 \\
\hline History of SGA & $1(1.2 \%)$ & 0 & 0 & 1 \\
\hline Group B streptococcus screen positive & $1(1.2 \%)$ & 0 & 0 & 1 \\
\hline \multicolumn{5}{|l|}{$N=22$ obstetrical antepartum covariates } \\
\hline \multicolumn{5}{|l|}{ Intrapartum or postpartum } \\
\hline Bishop score $<6$ & $1(1.2 \%)$ & 1 & 0 & 0 \\
\hline Unengaged fetal head & $3(3.7 \%)$ & 0 & 0 & 3 \\
\hline
\end{tabular}


Table 2 Risk factors tested for association with severe maternal morbidity (Continued)

\begin{tabular}{|c|c|c|c|c|}
\hline Potential covariates for use in risk adjustment & $\begin{array}{l}\text { Number of studies } \\
\text { that used the } \\
\text { variable in risk } \\
\text { adjustment }\end{array}$ & $\begin{array}{l}\text { Number of studies } \\
\text { with a statistically } \\
\text { significant result }\end{array}$ & $\begin{array}{l}\text { Number of } \\
\text { studies with a } \\
\text { non-significant } \\
\text { result }\end{array}$ & $\begin{array}{l}\text { Number of studies } \\
\text { where statistical } \\
\text { significance was not } \\
\text { reported }\end{array}$ \\
\hline Uterine rupture, prolapsed cord & $2(2.5 \%)$ & 0 & 0 & 3 \\
\hline Hemorrhage & $4(4.9 \%)$ & 1 & 0 & 3 \\
\hline Prior stillbirth or infant death & $4(4.9 \%)$ & 0 & 0 & 4 \\
\hline Stillbirth & $9(11.1 \%)$ & 0 & 0 & 9 \\
\hline $\begin{array}{l}\text { Route of delivery: includes labor } y / n \text {, operative } \\
V D^{q} \text { cesarean }\end{array}$ & $35(43.2 \%)$ & 12 & 3 & 20 \\
\hline Maternal indication for cesarean & $1(1.2 \%)$ & 0 & 0 & 1 \\
\hline Cesarean incision type & $1(1.2 \%)$ & 0 & 1 & 0 \\
\hline Induction & $12(14.8 \%)$ & 5 & 0 & 7 \\
\hline Cervical ripening & $1(1.2 \%)$ & 1 & 0 & 0 \\
\hline Epidural use & $3(3.7 \%)$ & 0 & 0 & 3 \\
\hline $\mathrm{PROM}^{\mathrm{r}} \mathrm{PPROM}^{\mathrm{s}}$ & $8(9.9 \%)$ & 0 & 0 & 8 \\
\hline Chorioamnionitis or maternal infection & $4(4.9 \%)$ & 1 & 0 & 3 \\
\hline Birth day: weekend, night & $6(7.4 \%)$ & 3 & 2 & 1 \\
\hline $\begin{array}{l}\text { Gestational age at delivery, preterm birth, type } \\
\text { of preterm birth }\end{array}$ & $33(40.7 \%)$ & 6 & 0 & 27 \\
\hline Preterm birth spontaneous vs indicated & $2(2.5 \%)$ & 0 & 0 & 2 \\
\hline $\begin{array}{l}\text { Labor anomalies: prolonged second stage, } \\
\text { oxytocin }\end{array}$ & $5(6.2 \%)$ & 1 & 0 & 4 \\
\hline General vs neuraxial anesthesia & $3(3.7 \%)$ & 2 & 0 & 1 \\
\hline Perineal trauma & $1(1.2 \%)$ & 0 & 0 & 1 \\
\hline Elective delivery & $6(7.4 \%)$ & 0 & 0 & 6 \\
\hline Fetal distress not in labor (separate from & $1(1.2 \%)$ & 0 & 0 & 1 \\
\hline
\end{tabular}

\section{$N=22$ intrapartum/postpartum covariates}

Other patient-level covariates

\begin{tabular}{|c|c|c|c|}
\hline Year of childbirth & $29(35.8 \%)$ & 13 & 2 \\
\hline Income & $2(2.5 \%)$ & 1 & 0 \\
\hline Rural & $4(4.9 \%)$ & 2 & 0 \\
\hline Insurance & $39(48.1 \%)$ & 16 & 4 \\
\hline Education & $21(25.9 \%)$ & 7 & 0 \\
\hline $\mathrm{SES}^{\mathrm{t}}$ & $5(6.2 \%)$ & 1 & 0 \\
\hline Foreign born & $16(19.8 \%)$ & 7 & 1 \\
\hline Language spoken & $1(1.2 \%)$ & 0 & 0 \\
\hline Refugee status & $1(1.2 \%)$ & 0 & 0 \\
\hline Duration of residence & $1(1.2 \%)$ & 0 & 0 \\
\hline Working & $2(2.5 \%)$ & 0 & 2 \\
\hline Married & $13(16.0 \%)$ & 3 & 2 \\
\hline Profession & $1(1.2 \%)$ & 0 & 0 \\
\hline Home birth & $1(1.2 \%)$ & 0 & 0 \\
\hline Transfer in from other hospital & $3(3.7 \%)$ & 0 & 0 \\
\hline
\end{tabular}

$N=15$ other patient-level covariates

Hospital-level covariates 
Table 2 Risk factors tested for association with severe maternal morbidity (Continued)

\begin{tabular}{|c|c|c|c|c|}
\hline Potential covariates for use in risk adjustment & $\begin{array}{l}\text { Number of studies } \\
\text { that used the } \\
\text { variable in risk } \\
\text { adjustment }\end{array}$ & $\begin{array}{l}\text { Number of studies } \\
\text { with a statistically } \\
\text { significant result }\end{array}$ & $\begin{array}{l}\text { Number of } \\
\text { studies with a } \\
\text { non-significant } \\
\text { result }\end{array}$ & $\begin{array}{l}\text { Number of studies } \\
\text { where statistical } \\
\text { significance was not } \\
\text { reported }\end{array}$ \\
\hline Hospital size or delivery volume & $17(21.0 \%)$ & 6 & 3 & 8 \\
\hline Hospitalist & $1(1.2 \%)$ & 0 & 1 & 0 \\
\hline Hospital ownership & $11(13.6 \%)$ & 4 & 2 & 5 \\
\hline Hospital teaching & $15(18.5 \%)$ & 5 & 2 & 8 \\
\hline Hospital urban/rural & $9(11.1 \%)$ & 2 & 2 & 5 \\
\hline Hospital percent high-risk & $1(1.2 \%)$ & 1 & 0 & 0 \\
\hline Hospital percent non-White & $1(1.2 \%)$ & 1 & 0 & 0 \\
\hline Hospital black-serving & $1(1.2 \%)$ & 1 & 0 & 0 \\
\hline Hospital percent Medicaid & $3(3.7 \%)$ & 1 & 0 & 2 \\
\hline Hospital coding intensity & $1(1.2 \%)$ & 1 & 0 & 0 \\
\hline Hospital percent midwife births & $1(1.2 \%)$ & 0 & 1 & 0 \\
\hline NICU ${ }^{u}$ level & $4(4.9 \%)$ & 2 & 1 & 1 \\
\hline $\begin{array}{l}\text { Hospital cesarean rate general endotracheal } \\
\text { anesthesia }\end{array}$ & $1(1.2 \%)$ & 0 & 0 & 1 \\
\hline Hospital epidural rate & $1(1.2 \%)$ & 0 & 0 & 1 \\
\hline Hospital induction rate & $1(1.2 \%)$ & 0 & 0 & 1 \\
\hline Hospital percent NTSV ${ }^{v}$ & $2(2.5 \%)$ & 0 & 0 & 2 \\
\hline Hospital percent early elective deliveries & $2(2.5 \%)$ & 0 & 0 & 2 \\
\hline Hospital Clinical Processes of Care quintiles & $1(1.2 \%)$ & 0 & 0 & 1 \\
\hline Hospital Patient Perspectives of Care quintiles & $1(1.2 \%)$ & 0 & 0 & 1 \\
\hline Hospital number triaged per day & $1(1.2 \%)$ & 0 & 0 & 1 \\
\hline Hospital number triaged per delivery & $1(1.2 \%)$ & 0 & 0 & 1 \\
\hline $\begin{array}{l}\text { Hospital > } 4 \text { hospitals within } 20 \text { miles of } \\
\text { residence }\end{array}$ & $1(1.2 \%)$ & 0 & 0 & 1 \\
\hline Hospital excellent doctor:nurse relationship & $1(1.2 \%)$ & 0 & 0 & 1 \\
\hline Hospital doctors/1000 deliveries & $1(1.2 \%)$ & 0 & 0 & 1 \\
\hline Hospital MFM ${ }^{w}$ on staff & $1(1.2 \%)$ & 0 & 0 & 1 \\
\hline Hospital midwives available & $1(1.2 \%)$ & 0 & 0 & 1 \\
\hline Hospital anesthesia available $24 / 7$ & $1(1.2 \%)$ & 0 & 0 & 1 \\
\hline $\begin{array}{l}\text { Hospital anesthesia staff have no other } \\
\text { responsibilities }\end{array}$ & $1(1.2 \%)$ & 0 & 0 & 1 \\
\hline Hospital equivalent staffing day and night & $1(1.2 \%)$ & 0 & 0 & 1 \\
\hline $\begin{array}{l}\text { Hospital cesarean in main hospital operating } \\
\text { room }\end{array}$ & $1(1.2 \%)$ & 0 & 0 & 1 \\
\hline Hospital radiology available $24 / 7$ & $1(1.2 \%)$ & 0 & 0 & 1 \\
\hline Hospital blood bank available24/7 & $1(1.2 \%)$ & 0 & 0 & 1 \\
\hline Hospital massive transfusion protocol in place & $1(1.2 \%)$ & 0 & 0 & 1 \\
\hline Hospital pharmacist dedicated to $L \& D^{x}$ & $1(1.2 \%)$ & 0 & 0 & 1 \\
\hline Hospital Bakri Balloon available & $1(1.2 \%)$ & 0 & 0 & 1 \\
\hline Hospital epidural easy to get & $1(1.2 \%)$ & 0 & 0 & 1 \\
\hline Hospital adult critical care $24 / 7$ & $1(1.2 \%)$ & 0 & 0 & 1 \\
\hline $\begin{array}{l}\text { Hospital subspecialty intensive care units } \\
\text { available }\end{array}$ & $1(1.2 \%)$ & 0 & 0 & 1 \\
\hline Hospital difficult to get consults & $1(1.2 \%)$ & 0 & 0 & 1 \\
\hline
\end{tabular}


Table 2 Risk factors tested for association with severe maternal morbidity (Continued)

\begin{tabular}{|c|c|c|c|c|}
\hline Potential covariates for use in risk adjustment & $\begin{array}{l}\text { Number of studies } \\
\text { that used the } \\
\text { variable in risk } \\
\text { adjustment }\end{array}$ & $\begin{array}{l}\text { Number of studies } \\
\text { with a statistically } \\
\text { significant result }\end{array}$ & $\begin{array}{l}\text { Number of } \\
\text { studies with a } \\
\text { non-significant } \\
\text { result }\end{array}$ & $\begin{array}{l}\text { Number of studies } \\
\text { where statistical } \\
\text { significance was not } \\
\text { reported }\end{array}$ \\
\hline Hospital has NICU & $1(1.2 \%)$ & 0 & 0 & 1 \\
\hline Hospital central FHR monitoring & $1(1.2 \%)$ & 0 & 0 & 1 \\
\hline $\begin{array}{l}\text { Hospital emergency response team available to } \\
\text { L\&D }\end{array}$ & $1(1.2 \%)$ & 0 & 0 & 1 \\
\hline Hospital allow TOLAC ${ }^{2}$ & $1(1.2 \%)$ & 0 & 0 & 1 \\
\hline $\begin{array}{l}\text { Hospital } 100 \% \text { of cesareans begun within } 30 \\
\text { min }\end{array}$ & $1(1.2 \%)$ & 0 & 0 & 1 \\
\hline $\begin{array}{l}\text { Hospital intermittent FHR monitoring }<50 \% \text { of } \\
\text { patients }\end{array}$ & $1(1.2 \%)$ & 0 & 0 & 1 \\
\hline Hospital doctors sign out to each other & $1(1.2 \%)$ & 0 & 0 & 1 \\
\hline Hospital formal rounds are conducted on L\&D & $1(1.2 \%)$ & 0 & 0 & 1 \\
\hline Hospital drills and simulations required & $1(1.2 \%)$ & 0 & 0 & 1 \\
\hline $\begin{array}{l}\text { Hospital FHR monitoring course required of } \\
\text { doctors }\end{array}$ & $1(1.2 \%)$ & 0 & 0 & 1 \\
\hline Hospital tracking of haemorrhage occurs & $1(1.2 \%)$ & 0 & 0 & 1 \\
\hline Hospital tracking of infection occurs & $1(1.2 \%)$ & 0 & 0 & 1 \\
\hline $\begin{array}{l}\text { Hospital tracking of } 3 \text { rd \& } 4 \text { th degree } \\
\text { lacerations occurs }\end{array}$ & $1(1.2 \%)$ & 0 & 0 & 1 \\
\hline Hospital has cesarean evaluation team & $1(1.2 \%)$ & 0 & 0 & 1 \\
\hline Hospital allows maternal transfers in & $1(1.2 \%)$ & 0 & 0 & 1 \\
\hline Hospital has a protocol for induction of labor & $1(1.2 \%)$ & 0 & 0 & 1 \\
\hline Hospital has a protocol for cesarean delivery & $1(1.2 \%)$ & 0 & 0 & 1 \\
\hline $\begin{array}{l}\text { Hospital gives education regarding induction of } \\
\text { labor }\end{array}$ & $1(1.2 \%)$ & 0 & 0 & 1 \\
\hline \multicolumn{5}{|l|}{$N=58$ hospital-level covariates } \\
\hline \multicolumn{5}{|l|}{ Community-level covariates } \\
\hline Region & $21(25.9 \%)$ & 6 & 1 & 14 \\
\hline Neighborhood poverty & $3(3.7 \%)$ & 0 & 2 & 1 \\
\hline Miles from zip code to hospital & $1(1.2 \%)$ & 0 & 1 & 0 \\
\hline Geographic designation of area urban/rural & $8(9.9 \%)$ & 2 & 0 & 6 \\
\hline $\begin{array}{l}\text { County frequency of obstetricians/ } \\
\text { anesthesiologists }\end{array}$ & $1(1.2 \%)$ & 0 & 1 & 0 \\
\hline County frequency of births to teens & $1(1.2 \%)$ & 0 & 1 & 0 \\
\hline County frequency of unmarried women & $1(1.2 \%)$ & 0 & 1 & 0 \\
\hline County frequency of divorced females & $1(1.2 \%)$ & 0 & 1 & 0 \\
\hline County frequency of female family heads & $1(1.2 \%)$ & 0 & 1 & 0 \\
\hline County frequency of females with no insurance & $1(1.2 \%)$ & 0 & 1 & 0 \\
\hline County frequency of foreign-born persons & $1(1.2 \%)$ & 0 & 1 & 0 \\
\hline $\begin{array}{l}\text { County frequency of persons with less than } \\
\text { high school education }\end{array}$ & $1(1.2 \%)$ & 0 & 1 & 0 \\
\hline County frequency of non-White persons & $1(1.2 \%)$ & 0 & 1 & 0 \\
\hline County household income measure & $18(22.2 \%)$ & 4 & 2 & 12 \\
\hline County frequency of unemployed persons & $1(1.2 \%)$ & 0 & 1 & 0 \\
\hline County frequency of food stamp beneficiaries & $1(1.2 \%)$ & 0 & 1 & 0 \\
\hline County frequency of persons with no phone & $1(1.2 \%)$ & 0 & 1 & 0 \\
\hline
\end{tabular}


Table 2 Risk factors tested for association with severe maternal morbidity (Continued)

\begin{tabular}{|c|c|c|c|c|}
\hline Potential covariates for use in risk adjustment & $\begin{array}{l}\text { Number of studies } \\
\text { that used the } \\
\text { variable in risk } \\
\text { adjustment }\end{array}$ & $\begin{array}{l}\text { Number of studies } \\
\text { with a statistically } \\
\text { significant result }\end{array}$ & $\begin{array}{l}\text { Number of } \\
\text { studies with a } \\
\text { non-significant } \\
\text { result }\end{array}$ & $\begin{array}{l}\text { Number of studies } \\
\text { where statistical } \\
\text { significance was not } \\
\text { reported }\end{array}$ \\
\hline $\begin{array}{l}\text { County frequency of households with }>1 \\
\text { person/room }\end{array}$ & $1(1.2 \%)$ & 0 & 1 & 0 \\
\hline County number of days with good air & $1(1.2 \%)$ & 0 & 1 & 0 \\
\hline County number of deaths due to AIDS aa & $1(1.2 \%)$ & 0 & 1 & 0 \\
\hline County number of deaths due to MVA ${ }^{\mathrm{bb}}$ & $1(1.2 \%)$ & 0 & 1 & 0 \\
\hline County death suicide & $1(1.2 \%)$ & 0 & 1 & 0 \\
\hline \multicolumn{5}{|l|}{$N=22$ community-level covariates } \\
\hline TOTAL: $N=180$ covariates & & & & \\
\hline
\end{tabular}

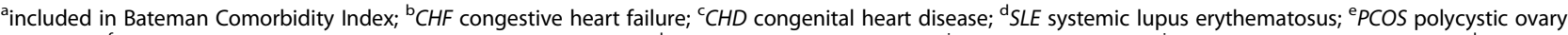
syndrome; ${ }^{\mathrm{f}} B M I$ body mass index; ${ }^{\mathrm{g} I B D}$ inflammatory bowel disease; ${ }^{\mathrm{h}} C N S$ central nervous system; ${ }^{\mathrm{i}} M S$ multiple sclerosis; ${ }^{\mathrm{j} V T E}$ venous thromboembolism; ${ }^{\mathrm{k}} / \mathrm{C} \mathrm{I}$ intrauterine insemination; 'ICSI intracytoplasmic sperm injection; ${ }^{\mathrm{m}} L G A$ large for gestational age; ${ }^{\mathrm{n}} S G A$ small for gestational age; ${ }^{\circ} P N C$ prenatal care; ${ }^{\mathrm{P}} D \& C$ dilatation and curettage; ${ }^{q} V D$ vaginal delivery; ${ }^{r} P R O M$ premature rupture of membranes; ${ }^{5} P P R O M$ preterm premature rupture of membranes; ${ }^{\mathrm{s}} S E S$ socioeconomic status; ${ }^{\mathrm{u}} N I C U$ neonatal intensive care unit; ${ }^{\mathrm{v}} N T S V$ nulliparous term singleton vertex; ${ }^{\mathrm{w}} M F M$ maternal fetal medicine specialist; ${ }^{\mathrm{x}} L \& D$ labor and delivery area; ${ }^{\mathrm{y}} F H R$ fetal heart rate; ${ }^{\mathrm{Z}} T O L A C$ trial of labor after cesarean; ${ }^{\text {aa }} \mathrm{AIDS}$ acquired immune deficiency syndrome; ${ }^{\mathrm{bb}} \mathrm{MVA}$ motor vehicle accident

may be attributable to multiple gestation; however, one publication found SMM risk to be elevated among singleton gestations [70].

There have been separate approaches to including drug, alcohol, and/or tobacco use as covariates in SMM models using administrative data. Some studies incorporated these conditions within a risk factor category labelled mental health while others treated these as separate risk factors. However, the sensitivity of administrative data for this information has been reported to be low $[96,97]$.

Fifteen studies examined specific intrapartum risk factors for their contribution to SMM: induction of labor $[40,65,82]$, off-hours delivery $[69,75]$, route of delivery $[20,24,41,56,66,74,77,84,94]$, and anesthesia type for cesarean delivery [16]. Others included intrapartum risk factors as covariates in the context of other hypotheses or in trying to explain the variation in SMM [21, 25, 37, 41, 46-48, 61, 63, 67, 76, 79]. Multiple investigators specifically used risk-adjustment models that only included antepartum risk factors for SMM to avoid adjustment for differences in patient management.

\section{Hypothesis testing of hospital-level risk factors for SMM}

Eight studies focused on hospital-level risk factors [19, 27, 29, 32, 36, 39, 49, 91]. There were few consistent findings. Three studies focused on annual hospital delivery volume and had mixed results [27, 29, 39]. Three other investigations tested various hypotheses regarding an association between the following specific hospital characteristics and SMM and found no association: the use of laborists in community hospitals [36], hospital quality indicators [49], and the percent of practitioners doing deliveries at the hospital that were midwives [19].
Given patients with the same high-risk conditions, it has been assumed that delivery at higher level hospitals will lead to less SMM. However, evidence for this supposition is limited. Two studies attempted to find an association between hospital resources and SMM. In both cases, hospital resource levels were studied as proxies for levels of maternal care, which are proposed designations for hospitals based on their resources and staffing [98]. Vanderlaan et al. used American Hospital Association data indicating the risk level of patients cared for by the hospital [91], and Clapp et al. assigned risk levels to patients based on Bateman's Obstetrical Comorbidity Index and then rated hospitals as high versus low acuity based on their percentages of high-risk patients [32]. In spite of extensive sensitivity analyses, Vanderlaan et al. found no relationship between these proxy resource levels and SMM [91]. Clapp et al. found that high-risk patients had a higher absolute risk of SMM at low-acuity hospitals when compared with high-risk patients at high-acuity centers; however, 95\% confidence intervals overlapped and no $p$-value for the comparison was reported [32].

\section{SMM rates and trends}

Twenty-seven of the included studies presented SMM rates. Several examined trends of SMM rates over the years $[17,26,38,45,72]$, reporting rising rates of both SMM and associated comorbidities. Some investigators disaggregated SMM rates and reported rates of the various indicators [18, 89]. SMM rates were highly dependent on the SMM definitions, study populations, and adjustment models. For example, some investigators built on the CDC definitions [52, 61-64]; others used broad definitions that included maternal intensive care unit admission [21, 25, 28, 40, 53, 65, 67, 71, 73]. A 
Table 3 Quality scoring of included studies $(n=81)$

\begin{tabular}{|c|c|c|c|c|c|c|}
\hline $\begin{array}{l}\text { REFERENCE } \\
\text { NUMBER }\end{array}$ & $\begin{array}{l}\text { LAST NAME OF FIRST } \\
\text { AUTHOR }\end{array}$ & YEAR & $\underset{* * *}{\text { SELECTION (MAX 3) }}$ & $\begin{array}{l}\text { RISK } \\
\text { FACTORS } \\
(\text { MAX 4) }\end{array}$ & $\underset{* * *}{\text { OUTCOME (MAX 3) }}$ & $\begin{array}{l}\text { TOTAL } \\
\text { SCORE }\end{array}$ \\
\hline [16] & $\mathrm{ABE}$ & 2018 & $* *$ & $* *$ & $*$ & 5 \\
\hline [17] & ADMON & 2018 & $* * *$ & $* *$ & * & 6 \\
\hline [18] & ADMON & 2018 & $* * *$ & $* *$ & $* *$ & 7 \\
\hline [19] & ATTANASIO & 2017 & * & $* *$ & * & 4 \\
\hline [20] & BANNISTER-TYRRELL & 2015 & $* *$ & $* *$ & * & 5 \\
\hline [21] & BARGER & 2013 & $* * *$ & $* *$ & * & 6 \\
\hline [22] & BATEMAN & 2013 & $* *$ & $* * *$ & $* *$ & 7 \\
\hline$[23]$ & BELANOFF & 2016 & $* * *$ & $* *$ & * & 6 \\
\hline$[24]$ & $\mathrm{BIN}$ & 2016 & $* *$ & $* *$ & * & 5 \\
\hline$[25]$ & BLANC & 2019 & * & $* * *$ & * & 5 \\
\hline [26] & BOOKER & 2018 & $* * *$ & $* * *$ & $* *$ & 8 \\
\hline [27] & BOOKER & 2018 & $* *$ & $* *$ & $* *$ & 6 \\
\hline [28] & BOURJEILY & 2017 & $* *$ & $* * *$ & * & 6 \\
\hline [29] & BOZZUTO & 2019 & $* * *$ & $* *$ & $*$ & 6 \\
\hline [30] & CHEN & 2015 & $* *$ & $* * *$ & * & 6 \\
\hline [31] & CHEN & 2013 & $* *$ & $* * *$ & * & 6 \\
\hline [32] & CLAPP & 2018 & $* * *$ & $* * *$ & $* *$ & 8 \\
\hline [33] & CREANGA & 2014 & $* * *$ & $* *$ & $* *$ & 7 \\
\hline [34] & DAYAN & 2019 & $* *$ & $* * *$ & $*$ & 6 \\
\hline [35] & DAYAN & 2018 & * & $* * *$ & * & 5 \\
\hline [36] & FELDMAN & 2015 & $* * *$ & $* * *$ & $*$ & 7 \\
\hline [37] & FORD & 2015 & $* *$ & $* *$ & $* *$ & 6 \\
\hline [38] & FREDERIKSEN & 2017 & $* * *$ & $* *$ & $* *$ & 7 \\
\hline [39] & FRIEDMAN & 2016 & $* * *$ & $* * *$ & $* *$ & 8 \\
\hline [40] & GIBBS PICKENS & 2018 & $* * *$ & $* *$ & $*$ & 6 \\
\hline [41] & GRASCH & 2017 & * & $* * *$ & * & 5 \\
\hline$[42]$ & GRAY & 2012 & $* * *$ & $* * *$ & $*$ & 7 \\
\hline [43] & GROBMAN & 2014 & $* * *$ & $* * * *$ & $* * *$ & 10 \\
\hline [44] & GUGLIELMINOTTI & 2019 & $* * *$ & $* *$ & $* *$ & 7 \\
\hline [45] & HEHIR & 2013 & $* *$ & $* * *$ & $* *$ & 7 \\
\hline$[46]$ & HOWELL & 2017 & $* *$ & $* *$ & $* *$ & 6 \\
\hline [47] & HOWELL & 2016 & $* *$ & $* * *$ & $* *$ & 7 \\
\hline [48] & HOWELL & 2016 & $* * *$ & $* *$ & * & 6 \\
\hline [49] & HOWELL & 2014 & $* *$ & $* * *$ & $* *$ & 7 \\
\hline [50] & HOWLAND & 2019 & $* *$ & $* * *$ & $* *$ & 7 \\
\hline [8] & JOSEPH & 2010 & $* *$ & $* *$ & * & 5 \\
\hline [51] & KILPATRICK & 2016 & $* * *$ & $* *$ & $* * *$ & 8 \\
\hline [52] & KORST & 2014 & $* * *$ & $* * *$ & $*$ & 7 \\
\hline [53] & LAZARIU & 2017 & $* * *$ & $* *$ & * & 6 \\
\hline [54] & LE RAY & 2019 & $* *$ & $* * *$ & $* * *$ & 8 \\
\hline [55] & LEONARD & 2019 & $* * *$ & $* * *$ & $* *$ & 8 \\
\hline [56] & LEONARD & 2019 & $* * *$ & $* * *$ & $* *$ & 8 \\
\hline [57] & LEONARD & 2019 & $* * *$ & $* * *$ & $* *$ & 8 \\
\hline
\end{tabular}


Table 3 Quality scoring of included studies $(n=81)$ (Continued)

\begin{tabular}{|c|c|c|c|c|c|c|}
\hline $\begin{array}{l}\text { REFERENCE } \\
\text { NUMBER }\end{array}$ & $\begin{array}{l}\text { LAST NAME OF FIRST } \\
\text { AUTHOR }\end{array}$ & YEAR & $\underset{* * *}{\text { SELECTION (MAX 3) }}$ & $\begin{array}{l}\text { RISK } \\
\text { FACTORS } \\
(\text { MAX 4) } * * * *\end{array}$ & $\underset{* * *}{\text { OUTCOME (MAX 3) }}$ & $\begin{array}{l}\text { TOTAL } \\
\text { SCORE }\end{array}$ \\
\hline [58] & LIESE & 2019 & $* * *$ & $* *$ & $* *$ & 7 \\
\hline [59] & LINDQUIST & 2015 & $* *$ & $* * *$ & * & 6 \\
\hline [60] & LIPKIND & 2019 & $* *$ & $* *$ & $* *$ & 6 \\
\hline [61] & LISONKOVA & 2016 & $* *$ & $* *$ & * & 5 \\
\hline [62] & LISONKOVA & 2017 & $* * *$ & $* * *$ & $*$ & 7 \\
\hline [63] & LISONKOVA & 2017 & $* * *$ & $* * *$ & * & 7 \\
\hline [64] & LISONKOVA & 2014 & $* * *$ & $* * *$ & $*$ & 7 \\
\hline$[65]$ & LIU & 2013 & $* *$ & * & $*$ & 4 \\
\hline [66] & LIU & 2007 & $* *$ & $* *$ & * & 5 \\
\hline [67] & LUKE & 2019 & $* * *$ & $* *$ & $*$ & 6 \\
\hline [68] & LYNDON & 2019 & $* * *$ & $* *$ & * & 6 \\
\hline [69] & LYNDON & 2015 & $* * *$ & $* *$ & $*$ & 6 \\
\hline [70] & MARTIN & 2016 & $* *$ & $* * *$ & * & 6 \\
\hline [71] & MASTERS & 2018 & $* * *$ & $* * *$ & $*$ & 7 \\
\hline [72] & METCALFE & 2018 & $* * *$ & $* * *$ & $*$ & 7 \\
\hline [73] & MOURAD & 2014 & $* *$ & $* *$ & * & 5 \\
\hline [74] & MURACA & 2019 & * & * & * & 3 \\
\hline [75] & NAM & 2019 & $* *$ & $* *$ & * & 5 \\
\hline [76] & OSMUNDSON & 2016 & $* * *$ & $* *$ & $*$ & 6 \\
\hline [77] & PALLASMAA & 2014 & $* *$ & $* *$ & * & 5 \\
\hline [78] & PLATNER & 2019 & $* *$ & $* * *$ & $* *$ & 7 \\
\hline [79] & RAMAGE & 2019 & $* * *$ & * & $*$ & 5 \\
\hline [80] & RAY & 2018 & $* *$ & $* * *$ & $*$ & 6 \\
\hline [81] & REID & 2018 & $* *$ & $* * *$ & $* *$ & 7 \\
\hline [82] & ROBERTS & 2009 & $* *$ & $* *$ & * & 5 \\
\hline [83] & ROSENBLOOM & 2017 & $* *$ & $* * * *$ & * & 7 \\
\hline [84] & ROY & 2019 & $* * *$ & $* *$ & $*$ & 6 \\
\hline [85] & SCHUMMERS & 2018 & $* *$ & $* * * *$ & $*$ & 7 \\
\hline [86] & SCHUMMERS & 2015 & $* *$ & $* * * *$ & $* *$ & 8 \\
\hline [87] & SHAND & 2016 & $* *$ & $* * *$ & * & 6 \\
\hline [88] & SIDDIQUI & 2019 & $* *$ & $* * * *$ & $* *$ & 8 \\
\hline [89] & URQUIA & 2017 & $* *$ & * & * & 4 \\
\hline [90] & URQUIA & 2015 & * & * & $* *$ & 4 \\
\hline [91] & VANDERLAAN & 2019 & $* * *$ & $* * *$ & $* *$ & 8 \\
\hline [92] & WAHLBERG & 2013 & $* *$ & $* * *$ & $* *$ & 7 \\
\hline [93] & WANG & 2016 & & $* * * *$ & $* * *$ & 7 \\
\hline [3] & WEN & 2005 & $* *$ & $* * *$ & * & 6 \\
\hline [95] & YOUNG & 2018 & $* *$ & $* *$ & $*$ & 5 \\
\hline
\end{tabular}

number of studies extended SMM case finding to 42 days postpartum or readmission with SMM.

In the last 5 years, and particularly with the use of administrative data wherein the number of units of packed red blood cells cannot be reliably ascertained, investigators have recognized that blood transfusion accounts for a large proportion of the SMM cases, and, consequently, whether or not it is included in the SMM definition substantially affects the SMM rate and its interpretability [7]. Fifteen studies did sensitivity analyses 
to display trends or determine if the effect sizes of risk factors were confirmed when transfusion was eliminated from the SMM definition. Trends from year to year were less likely to show statistical differences, and most studies (with some exceptions [50, 55, 78, 94] showed minimal to no changes in the magnitude of risk factors when excluding transfusion. Another 10 did not include transfusion in their SMM definition, nine studies using a maternal ICU admission did not separate transfusion out, and six used medical chart review to assure that at least 4 units of packed red blood cells were used to qualify as meeting the SMM definition (Table 1).

From the seven studies using administrative data with unrestricted delivery populations and including transfusion in the SMM definition [3, 8, 38, 44, 53, 60, 81], SMM rates varied from $0.44 \%$ [3] to $2.55 \%$ [53]. Using the US National Inpatient Sample [99], the CDC reported the most recent SMM rates from 2014 as $1.44 \%$ with transfusions and $0.35 \%$ when using the definition excluding transfusions [7]. The overall rate of SMM increased 200\% from 1993 to 2014 when transfusion was included and $20 \%$ in the same time period when transfusion was excluded.

Maternal death is not an exclusion criterion for the CDC definition of SMM [7]. Some studies specifically included maternal death whether or not SMM was reported. One posited that the coding of death without SMM must be erroneous, and, therefore, excluded such cases [70]. Friedman et al. studied both SMM and death, finding that: 1) $78.7 \%$ of deaths in the dataset had been identified as having SMM (these deaths were referred to as "failure to rescue"); and 2) $1.0 \%$ of patients with SMM died [39]. This study did not extend the SMM definition to include post-discharge follow-up. In a study by Ray et al., $68.0 \%$ of deaths in a population-based delivery cohort had been identified as having SMM [80].

\section{Discussion \\ Principal findings}

This review identified 81 studies of SMM that relied on risk adjustment of routinely collected population-based data. Although the key search term was deliberately chosen to be "severe maternal morbidity" in an attempt to identify studies that incorporated similar outcomes, only $37(45.7 \%)$ used an SMM definition with a CDC basis; the SMM definitions used in the remaining studies varied to a much larger extent. The inclusion of blood transfusions (yes/no) in the SMM definition added a layer of complexity to the comparability of these analyses, given that, in various studies, more than half of the SMM cases had this single indicator of SMM. Such heterogeneity was also evident in the principal datasets used (e.g., claims data, electronic medical record or medical record data, administrative data in both ICD-9 and ICD-
10), which may have included linkages to other datasets (e.g., infertility, birth certificate, hospital surveys, census data). Study populations also differed with respect to the definition of a delivery admission and, depending on the purpose of the study, the inclusion and exclusion criteria.

The covariates used for risk adjustment also varied extensively ( $n=180$, Table 2 ), not only with respect to the choice of covariates, but also with respect to their definitions (e.g., BMI as a continuous, ordinal, or binary variable). Interpretation of the results also depends on the study design (e.g., subset of deliveries included) and model specification (e.g., other covariates included). Some studies attempted to limit the types of covariates to patient-level conditions that would be apparent prior to the childbirth admission, while others attempted to develop more explanatory models for SMM and included intrapartum variables such as dystocia and delivery mode. Several studies used hospital characteristics (e.g., delivery volume, ownership, or teaching status) or community-level variables (e.g., median household income, percent foreign-born by zip code or county) to make comparisons more interpretable or models more explanatory. Consequently, effect sizes (odds ratios and relative risks) could not be synthesized in a meaningful way.

\section{Interpretation}

The call for facility-based surveillance of SMM through case review $[10,11]$ remains critical for identifying SMM causes, so that prevention strategies and interventions can be developed, implemented, and tested prospectively. In addition, there remains a role for populationbased administrative data to describe and monitor the SMM burden [12]. The use of administrative data enables the development of standard SMM rates that can be used to describe trends and disparities and, potentially, to make comparisons across regions and hospitals. Such comparisons can highlight regions or hospitals with disproportionate burdens and can potentially provide insight regarding the quality of pregnancy care for those with SMM and/or the resources needed to address SMM. This use of administrative data at the population level can also inform decisions regarding the potential for public health interventions, such as improving the availability of preconception care [100], and can be used to track their success. Demonstrated success could mean more resources can be deployed to scale-up effective interventions and attenuate the SMM burden.

The results of this review point to several areas that are in need of development for the continued evolution of SMM tracking using population-based data. First is the standardization of the SMM definition. In the US, this definition has been gravitating toward that used by 
the CDC. However, differences remain across recent US studies, particularly with respect to the inclusion of blood transfusion. The role of transfusions in the administrative definition of SMM needs further evaluation and standardization because the rise in transfusions is due largely to quality improvement efforts to decrease mortality from postpartum hemorrhage [101]. It is apparent that blood products are increasingly being used as part of a secondary prevention effort and that such usage in practice (which is lifesaving) conflicts with the interpretation of the SMM measure as a poor outcome.

The second area in need of development is the standardization of the content and size of datasets used for hospital or regional comparisons. Hospital discharge datasets appear to be the best choice because they are relatively similar and nearly universally available. The marginal benefit for the addition of linked patient-level datasets, such as the birth certificate data, may be too resource-intensive for some states. The linkage of a basic subset of hospital variables such as ownership, delivery volume, and teaching status, could be gleaned from a variety of sources and maintained in a central location for consistent use. The importance of community-level variables (e.g., by census tract, zip code, county) has not been well-explored in the literature and needs further evaluation, especially as it relates to the potential for public health intervention and ability to impact SMM rates. Community-level summary measures (e.g., median income, rural status) were frequently used as proxies for patient- or hospital-level comparisons and were relatively infrequently reported as contributing to risk adjustment models.

Third is the selection and definition of risk factors of interest. This will depend on the purpose of the risk adjustment. For the purpose of comparing hospital SMM rates, we suggest that models should adjust for case-mix using the risk factors known upon admission but without including those variables describing intrapartum management (e.g., route of delivery) because these variables are under the control of a given hospital and there is no need to keep them balanced across hospitals. Hospital-level factors, such as resources or staffing characteristics, should also be excluded if hospitals are being ranked. The "within" hospital correlation in SMM can be addressed using clustered standard errors. A more serviceable comparison can be achieved by comparing only hospitals of the same type (e.g., teaching hospitals or community hospitals). By confining hospital comparisons to a group with a similar type, the average hospital for that type yields a better representation of the group compared with an average hospital in a group composed of diverse hospital types. On the other hand, if the purpose is to predict the SMM risk, it is reasonable for these models to include intrapartum-, hospital-, and community-level risk factors to increase explanatory power.

Furthermore, the inclusion of patient-level non-clinical variables (e.g., insurance type, educational level) in SMM risk adjustment models deserves reflection. Such variables may be potential proxies for unmeasured clinical risk factors (e.g., malnutrition), measures of access to higher quality of care, or sources of variation due to discrimination. The risk adjustment purpose and the hypothesized source for the variation in SMM risk due to such variables should determine their use in modelling. For example, for hospital comparisons, use of these covariates would not be appropriate given that they would credit hospitals for poor care given to disadvantaged patients.

\section{Limitations of the review}

As discussed in depth above, a limitation of this review is the study heterogeneity, which prevents meaningful synthesis of effect sizes. More narrow inclusion criteria may have allowed for increased detail regarding the relative importance of specific risk factors, such as race/ethnicity and prematurity.

\section{Conclusions}

This review identified multiple potential risk factors associated with SMM. The heterogeneity of the studies precluded any quantitative synthesis of the effect sizes (odds ratios or relative risks) of the identified risk factors. The development of a risk adjustment strategy that will allow for SMM comparisons across hospitals or regions will require harmonization regarding the standardization of the SMM definition, the datasets and population used, and the selection and definition of risk factors of interest. The ability to perform such comparisons would contribute to the capacity of public health systems to monitor SMM trends and disparities and to develop strategies to decrease the SMM burden. Administrative data comparisons also allow for evaluating the potential for interventions, tracking their success, and estimating the resources needed to scale-up effective interventions.

\section{Supplementary Information}

The online version contains supplementary material available at https://doi. org/10.1186/s40748-020-00123-1.

Additional file 1. Newcastle-Ottawa Quality Assessment Scale (adapted
for cross sectional studies with healthcare data for research question:
What are the risk factors for severe maternal morbidity associated with
the delivery admission?
Additional file 2. Preferred Reporting Items for Systematic reviews and Additional file 2. Preferred Reporting Items for Systematic reviews and
Meta-Analyses Extension for Scoping Reviews (PRISMA-SCR) Checklist. 


\section{Abbreviations}

AIDS: Acquired immune deficiency syndrome; ART: Assisted reproductive technology; BMI: Body mass index; CDC: Centers for Disease Control and Prevention; CHF: Congestive heart failure; CHD: Congenital heart disease: CNS: Central nervous system; CMS: Centers for Medicare and Medicaid Services; D\&C: Dilatation and curettage; FHR: Fetal heart rate; IBD: Inflammatory bowel disease; ICD-9, 10: International Classification of Diseases, Clinical Modification, versions 9,10; ICSI: Intracytoplasmic sperm injection; IUI: Intrauterine insemination; IVF: In vitro fertilization; L\&D: Labor and delivery area; LGA: Large for gestational age; MFM: Maternal fetal medicine specialist; MS: Multiple sclerosis; MVA: Motor vehicle accident; NICU: Neonatal intensive care unit; NOS: Newcastle-Ottawa Scale; NTSV: Nulliparous term singleton vertex; PCOS: Polycystic ovary syndrome; PNC: Prenatal care; PRISMA-ScR: Preferred Reporting Items for Systematic Reviews and Meta-analyses extension for scoping reviews; PROM: Premature rupture of membranes; PPROM: Preterm premature rupture of membranes; SART: Society for Assisted Reproductive Technology; SES: Socioeconomic status; SGA: Small for gestational age; SLE: Systemic lupus erythematosus; SMM: Severe maternal morbidity; TOLAC : Trial of labor after cesarean; VD: Vaginal delivery; VTE: Venous thromboembolism; US: United States; WHO: World Health Organization

\section{Acknowledgments}

None.

\section{Authors' contributions}

Conceptualization: LMK,KDG,LAN,SS,DJR,JLT,NG,MF; Data curation: LMK,MF; Formal analysis: LMK, MF; Funding acquisition: LMK,KDG,LAN,DJR,JLT,MF; Investigation: LMK,KDG,LAN,SS,DJR,JLT,NG,MF; Methodology: LMK,KDG,NG,MF; Project administration: LAN, KDG,SS,DJR,JLT; Resources: LMK,MF; Supervision: KDG,LAN,DJR,MF; Validation: LMK, MF; Visualization: LMK,KDG,LAN,DJR,JLT,MF; Writing-original draft preparation: LMK, MF; Writing-review and editing: $L M K, K D G, L A N, S S, D J R, J L T, N G, M F$. The authors read and approved the final manuscript.

\section{Authors' information}

Dr. Kimberly Gregory is Helping Hand of Los Angeles Mariam Jacobs Chair in Maternal Fetal Medicine in the Department of Obstetrics and Gynecology at Cedars-Sinai Medical Center.

\section{Funding}

Funding source: California Department of Public Health, Maternal, Child and Adolescent Health Division, contract number 18-10003. (LMK, KDG, LAN, and MF received the award.) role of the sponsor: DJR and JLT were involved in the study design, data collection and analysis, decision to publish, and preparation of the manuscript. The original funding source for this award is the Title V Maternal and Child Health Services Block Grant from the Maternal and Child Health Bureau/Health Resources \& Services Administration.

\section{Availability of data and materials}

No data or materials were used for the publication of this article beyond the review of those articles referenced.

\section{Ethics approval and consent to participate}

Not applicable.

\section{Consent for publication}

Not applicable.

\section{Competing interests}

The authors report no conflicts of interest and the following disclosures. LMK and MF are shareholders in Maternal Metrics, Inc. LMK, MF, KDG, and LAN are recipients of federal and state grants for work regarding obstetrical outcomes. DJR and JLT are employees of the California Department of Public Health (CDPH). The remaining authors have nothing to disclose.

\section{Author details}

'Childbirth Research Associates, LLC, North Hollywood, CA, USA. ${ }^{2}$ Department of Obstetrics and Gynecology, Cedars-Sinai Medical Center, Burns Allen Research Institute, Los Angeles, CA, USA. ${ }^{3}$ Department of Obstetrics and Gynecology, David Geffen School of Medicine at UCLA, Los
Angeles, CA, USA. ${ }^{4}$ Department of Community Health Sciences, Fielding School of Public Health at UCLA, Los Angeles, CA, USA. ${ }^{5}$ Maternal, Child and Adolescent Health Division, California Department of Public Health, Sacramento, CA, USA. ${ }^{6}$ AMF Consulting, Los Angeles, CA, USA.

Received: 15 September 2020 Accepted: 9 December 2020

Published online: 06 January 2021

\section{References}

1. World Health Organization. Beyond the numbers: reviewing maternal deaths and complications to make pregnancy safer. Geneva: World Health Organization; 2004. Available from: https://apps.who.int/iris/handle/10665/42 984. Accessed 1 Sept 2020.

2. Say L, Souza JP, Pattinson RC, for the WHO working group on Maternal Mortality and Morbidity classifications. Maternal near miss - towards a standard tool for monitoring quality of maternal health care. Best Pract Res Clin Obstet Gynaecol. 2009;23:287-96.

3. Wen SW, Huang L, Liston R, Heaman M, Baskett T, Rusen ID, et al. Severe maternal morbidity in Canada, 1991-2001. CMAJ. 2005;173(7):759-64.

4. Callaghan WM, MacKay AP, Berg CY. Identification of severe maternal morbidity during delivery hospitalizations, United States, 1991-2003. Am J Obstet Gynecol. 2008;199:133.e1-8.

5. Callaghan WM, Creanga AA, Kuklina EV. Severe maternal morbidity among delivery and postpartum hospitalizations in the United States. Obstet Gynecol. 2012;120:1029-36.

6. Centers for Disease Control and Prevention. How does CDC identify severe maternal morbidity. Available from: https://www.cdc.gov/ reproductivehealth/maternalinfanthealth/smm/severe-morbidity-ICD.htm. Accessed 1 Sept 2020.

7. Roberts $\mathrm{CL}$, Cameron CA, Bell JC, Algert CS, Morris JM. Measuring maternal morbidity in routinely collected health data: development and validation of a maternal morbidity outcome indicator. Med Care. 2008;46(8):786-94.

8. Joseph KS, Liu S, Rouleau J, Kirby RS, Dramer MS, Sauve R, et al. Severe maternal morbidity in Canada, 2003 to 2007: surveillance using routine hospitalization data and ICD-10ca codes. J Obstet Gynaecol Can. 2010;32(9): 837-46

9. Callaghan WM, Grobman WA, Kilpatrick SJ, Main EK, D'Alton M. Facilitybased identification of women with severe maternal morbidity: it is time to start. Obstet Gynecol. 2014;123:978-81.

10. Kilpatrick SJ, Berg C, Bernstein P, Bingham D, Delgado A, Callaghan WM, et al. Standardized severe maternal morbidity review: rationale and process. Obstet Gynecol. 2014;124:361-6.

11. Kuklina EV, Goodman DA. Severe maternal or near miss morbidity: implications for public health surveillance and clinical audit. Clin Obstet Gynecol. 2018;61(2):307-18.

12. National Quality Forum. National Quality Forum launches new project to improve maternal morbidity and mortality outcomes. 2019. Available from: https://www.pressreleasepoint.com/national-quality-forum-launches-newproject-improve-maternal-morbidity-and-mortality-outcomes. Accessed 1 Sept 2020.

13. Anderson S, Allen P, Peckham S, Goodwin N. Asking the right questions: scoping studies in the commissioning of research on the organization and delivery of health services. Health Res Policy Sys. 2008;6:7.

14. Preferred Reporting Items for Systematic Reviews and Meta-Analyses (PRIS MA) website. Available from: http://www.prisma-statement.org/Extensions/ ScopingReviews. Accessed 1 Sept 2020.

15. Wells GA, Shea B, O'Connell D, Robertson J, Peterson J, Welch $V$ et al. The Newcastle-Ottawa scale (NOS) for assessing the quality of nonrandomised studies in meta-analyses. Available from: http://www.ohri.ca/programs/ clinical_epidemiology/oxford.asp. Accessed 1 Sept 2020.

16. Abe H, Sumitani M, Uchida K, Ikeda T, Matsui H, Fushimi K, et al. Association between mode of anaesthesia and severe maternal morbidity during admission for scheduled caesarean delivery: a nationwide population-based study in Japan, 2010-2013. Br J Anaesth. 2018;120(4):779-89.

17. Admon LK, Bart G, Kozhimannil KB, Richardson CR, Dalton VK, Winkelman TNA. Amphetamine- and opioid-affected births: incidence, outcomes, and costs, United States, 2004-2015. Am J Public Health. 2018;109(1):148-54.

18. Admon LK, Winkelman TNA, Zivin K, Terplan M, Mhyre JM, Dalton VK. Racial and ethnic disparities in the incidence of severe maternal morbidity in the United States, 2012-2015. Obstet Gynecol. 2018;132(5):1158-66. 
19. Attanasio L, Kozhimannil KB. Relationship between hospital-level percentage of midwife-attended births and obstetric procedure utilization. J Midwifery Womens Health. 2018;63(1):14-22.

20. Bannister-Tyrrell M, Patterson JA, Ford JB, Morris JM, Nicholl MC, Roberts CL. Variation in hospital caesarean section rates for preterm births. Aust N Z J Obstet Gynaecol. 2015;55(4):350-6.

21. Barger MK, Nannini A, DeJoy S, Wisner K, Markenson G. Maternal and newborn outcomes following uterine rupture among women without versus those with a prior cesarean. J Matern Fetal Neonatal Med. 2013;26(2): 183-7.

22. Bateman BT, Mhyre JM, Hernandez-Diaz S, Huybrechts KF, Fischer MA, Creanga AA, et al. Development of a comorbidity index for use in obstetric patients. Obstet Gynecol. 2013;122(5):957-65.

23. Belanoff C, Declercq ER, Diop H, Gopal D, Kotelchuck M, Luke B, et al. Severe maternal morbidity and the use of assisted reproductive technology in Massachusetts. Obstet Gynecol. 2016;127(3):527-34.

24. Bin YS, Roberts CL, Ford JB, Nicholl MC. Outcomes of breech birth by mode of delivery: a population linkage study. Aust N Z J Obstet Gynaecol. 2016; 56(5):453-9.

25. Blanc JN, Resseguier JN, Goffinet F, Lorthe E, Kayem G, Delorme P, et al. Association between gestational age and severe maternal morbidity and mortality of preterm cesarean delivery: a population-based cohort study. Am J Obstet Gynecol. 2019;220(4):399.e1-9.

26. Booker WA, Gyamfi-Bannerman C, Sheen JJ, Wright JD, Siddiq Z, D'Alton ME, et al. Maternal outcomes by race for women aged 40 years or older. Obstet Gynecol. 2018;132(2):404-13

27. Booker WA, Ananth CV, Wright JD, Siddiq A, D'Alton ME, Cleary KL, et al. Trends in comorbidity, acuity, and maternal risk associated with preeclampsia across obstetric volume settings. J Matern Fetal Neonatal Med. 2018;32(16):2680-7.

28. Bourjeily G, Danilack VA, Bublitz MH, Lipkind H, Muri J, Caldwell D, et al. Obstructive sleep apnea in pregnancy is associated with adverse maternal outcomes: a national cohort. Sleep Med. 2017;38:50-7.

29. Bozzuto L, Passarella M, Lorch S, Srinivas S. Effects of delivery volume and high-risk condition volume on maternal morbidity among high-risk obstetric patients. Obstet Gynecol. 2019;133(2):261-8.

30. Chen JS, Roberts CL, Simpson JM, March LM. Pregnancy outcomes in women with rare autoimmune diseases. Arthritis Rheumatol. 2015;67(12): 3314-23.

31. Chen JS, Ford JB, Roberts CL, Simpson JM, March LM. Pregnancy outcomes in women with juvenile idiopathic arthritis: a population-based study. Rheumatology. 2013;52(6):1119-25.

32. Clapp MA, James KE, Kaimal AJ. The effect of hospital acuity on severe maternal morbidity in high-risk patients. Am J Obstet Gynecol. 2018;219(1): 111.e1-7.

33. Creanga AA, Bateman BT, Kuklina EV, Callaghan WM. Racial and ethnic disparities in severe maternal morbidity: a multistate analysis, 2008-2010. Am J Obstet Gynecol. 2014;210(5):435.e1-8.

34. Dayan N, Joseph KS, Fell DB, Laskin CA, Basso O, Park AL, et al. Infertility treatment and risk of severe maternal morbidity: a propensity scorematched cohort study. CMAJ. 2019;191(5):e118-27.

35. Dayan N, Fell DB, Guo Y, Wang H, Velez MP, Spitzer K, et al. Severe maternal morbidity in women with high BMI in IVF and unassisted singleton pregnancies. Hum Reprod. 2018;33(8):1548-56.

36. Feldman DS, Bollman DL, Fridman M, Korst LM, El Haj IS, Fink A, et al. Do laborists improve delivery outcomes for laboring women in California community hospitals? Am J Obstet Gynecol. 2015;213(4):587.e581-7.

37. Ford JB, Patterson JA, Seeho SK, Roberts CL. Trends and outcomes of postpartum haemorrhage, 2003-2011. BMC Pregnancy Childbirth. 2015;15: 334.

38. Frederiksen BN, Lillehoj CJ, Kane DJ, Goodman D, Rankin K. Evaluating lowa severe maternal morbidity trends and maternal risk factors: 2009-2014. Matern Child Health J. 2017;21(9):1834-44.

39. Friedman AM, Ananth CV, Huang Y, D'Alton ME, Wright JD. Hospital delivery volume, severe obstetrical morbidity, and failure to rescue. Am J Obstet Gynecol. 2016;215(6):795.e1-795.e714.

40. Gibbs Pickens CM, Kramer MR, Howards PP, Badell ML, Caughey AB, Hogue CJ. Term elective induction of labor and pregnancy outcomes among obese women and their offspring. Obstet Gynecol. 2018;131(1): $12-22$.
41. Grasch JL, Thompson JL, Newton JM, Zhai AW, Osmundson SS. Trial of labor compared with cesarean delivery in superobese women. Obstet Gynecol. 2017;130(5):994-1000.

42. Gray KE, Wallace ER, Nelson KR, Reed SD, Schiff MA. Population-based study of risk factors for severe maternal morbidity. Paediatr Perinat Epidemiol. 2012;26(6):506-14.

43. Grobman WA, Bailit JL, Rice MM, Wapner RJ, Reddy UM, Varner MW, et al. Frequency of and factors associated with severe maternal morbidity. Obstet Gynecol. 2014;123(4):804-10.

44. Guglielminotti J, Landau R, Wong CA, Li G. Patient-, hospital-, and neighborhood-level factors associated with severe maternal morbidity during childbirth: a cross-sectional study in New York state 2013-2014. Matern Child Health J. 2019:23(1):82-91.

45. Hehir MP, Ananth CV, Wright JD, Siddiq Z, D'Alton ME, Friedman AM. Severe maternal morbidity and comorbid risk in hospitals performing $<1000$ deliveries per year. Am J Obstet Gynecol. 2017;216(2):179.e1-179.e12.

46. Howell EA, Egorova NN, Janevic T, Balbierz A, Zeitlin J, Hebert PL. Severe maternal morbidity among Hispanic women in New York City: investigation of health disparities. Obstet Gynecol. 2017;129(2):285-94

47. Howell EA, Egorova NN, Balbierz A, Zeitlin J, Hebert PL. Site of delivery contribution to black-white severe maternal morbidity disparity. Am J Obstet Gynecol. 2016;215(2):143-52.

48. Howell EA, Egorova N, Balbierz A, Zeitlin J, Hebert PL. Black-white differences in severe maternal morbidity and site of care. Am J Obstet Gynecol. 2016;214(1):122.e1-7.

49. Howell EA, Zeitlin J, Hebert PL, Balbierz A, Egorova N. Association between hospital-level obstetric quality indicators and maternal and neonatal morbidity. JAMA. 2014;312(15):1531-41.

50. Howland RE, Angley M, Won SH, Wilcox W, Searing H, Liu SY, et al. Determinants of severe maternal morbidity and its racial/ethnic disparities in New York City, 2008-2012. Matern Child Health J. 2019;23(3):346-55.

51. Kilpatrick SJ, Abreo A, Gould J, Greene N, Main EK. Confirmed severe maternal morbidity is associated with high rate of preterm delivery. Am J Obstet Gynecol. 2016;215(2):233.e1-7.

52. Korst LM, Fridman M, Lu MC, Mitchell C, Lawton E, Griffin F, et al. Monitoring childbirth morbidity using hospital discharge data: further development and application of a composite measure. Am J Obstet Gynecol. 2014;211(3):268.e1-268.e16.

53. Lazariu V, Nguyen T, McNutt LA, Jeffrey J, Kacica M. Severe maternal morbidity: a population-based study of an expanded measure and associated factors. PLoS One. 2017;12(8):e0182343.

54. Le Ray C, Pelage L, Seco A, Bouvier-Colle MH, Chantry AA, DeneuxTharaux $C$, et al. Risk of severe maternal morbidity associated with in vitro fertilisation: a population-based study. BJOG. 2019:126(8):1033-41.

55. Leonard SA, Carmichael SL, Main EK, Lyell DJ, Abrams B. Risk of severe maternal morbidity in relation to prepregnancy body mass index: roles of maternal co-morbidities and caesarean birth. Paediatr Perinat Epidemiol. 2020;34(4):460-8

56. Leonard SA, Main EK, Carmichael SL. The contribution of maternal characteristics and cesarean delivery to an increasing trend of severe maternal morbidity. BMC Pregnancy Childbirth. 2019;19(1):16.

57. Leonard SA, Main EK, Scott KA, Profit J, Carmichael SL. Racial and ethnic disparities in severe maternal morbidity prevalence and trends. Ann Epidemiol. 2019:33:30-6.

58. Liese KL, Mogos M, Abboud S, Decocker K, Koch AR, Geller SE. Racial and ethnic disparities in severe maternal morbidity in the United States. J Racial Ethn Health Disparities. 2019;6(4):790-8.

59. Lindquist AC, Kurinczuk JJ, Wallace EM, Oats J, Knight M. Risk factors for maternal morbidity in Victoria, Australia: a population-based study. BMJ Open. 2015:5(8):e007903.

60. Lipkind HS, Zuckerwise LC, Bragan Turner E, Collins JJ, Campbell KH, Reddy UM, et al. Severe maternal morbidity during delivery hospitalization in a large international administrative database, 2008-2013: a retrospective cohort. BJOG. 2019;126(10):1223-30.

61. Lisonkova S, Haslam MD, Dahlgren L, Chen I, Synnes AR, Lim Kl. Maternal morbidity and perinatal outcomes among women in rural versus urban areas. CMAJ. 2016:188(17-18):e456-65.

62. Lisonkova S, Muraca GM, Potts J, Liauw J, Chan WS, Skoll A, et al. Association between prepregnancy body mass index and severe maternal morbidity. JAMA. 2017;318(18):1777-86. 
63. Lisonkova S, Potts J, Muraca GM, Razaz N, Sabr Y, Chan WS, et al. Maternal age and severe maternal morbidity: a population-based retrospective cohort study. PLoS Med. 2017;14(5):e1002307.

64. Lisonkova S, Sabr Y, Mayer C, Young C, Skoll A, Joseph KS. Maternal morbidity associated with early-onset and late-onset preeclampsia. Obstet Gynecol. 2014;124(4):771-81.

65. Liu S, Liston RM, Joseph KS, Heaman M, Sauve R, Kramer MS. Gestational age-specific severe maternal morbidity associated with labor induction. Am J Obstet Gynecol. 2013;209(3):209.e1-8.

66. Liu S, Liston RM, Joseph KS, Heaman M, Sauve R, Kramer MS. Maternal mortality and severe morbidity associated with low-risk planned cesarean delivery versus planned vaginal delivery at term. CMAJ. 2007;176(4):455-60

67. Luke B, Baker VL, Doody KJ. Risk of severe maternal morbidity by maternal fertility status: a US study in 8 states. Am J Obstet Gynecol. 2019;220(2):195. e1-195.e12.

68. Lyndon A, Baer RJ, Gay CL, El Ayadi AM, Lee HC, Jelliffe-Pawlowski L. A population-based study to identify the prevalence and correlates of the dual burden of severe maternal morbidity and preterm birth in California. J Matern Fetal Neonatal Med. 2019. https://doi.org/10.1080/14767058.2019. 1628941.

69. Lyndon A, Lee HC, Gay C, Gilbert WM, Gould JB, Lee KA. Effect of time of birth on maternal morbidity during childbirth hospitalization in California. Am J Obstet Gynecol. 2015;213(5):705.e1-11.

70. Martin AS, Monsour M, Kissin DM, Jamieson DJ, Callaghan WM, Boulet SL. Trends in severe maternal morbidity after assisted reproductive technology in the United States, 2008-2012. Obstet Gynecol. 2016;127(1):59-66.

71. Masters HR, Housley E, van Hook JW, DeFranco E. Maternal obesity is an independent risk factor for intensive care unit admission during delivery hospitalization. Am J Perinatol. 2018;35(14):1423-8.

72. Metcalfe A, Wick J, Ronksley P. Racial disparities in comorbidity and severe maternal morbidity/mortality in the United States: an analysis of temporal trends. Acta Obstet Gynecol Scand. 2018;97(1):89-96.

73. Mourad M, Silverstein M, Bender S, Melka S, Klauser CK, Gupta S, et al. The effect of maternal obesity on outcomes in patients undergoing tertiary or higher cesarean delivery. J Matern Fetal Neonatal Med. 2015;28(9):989-93.

74. Muraca GM, Sabr Y, Lisonkova S, Skoll A, Brant R, Cundiff GW, et al. Morbidity and mortality associated with forceps and vacuum delivery at outlet, low, and midpelvic station. J Obstet Gynaecol Can. 2019;41(3):32737.

75. Nam JY, Lee SG, Nam CM, Park S, Jang SI, Park EC. The effect of off-hour delivery on severe maternal morbidity: a population-based cohort study. Eur J Pub Health. 2019;29(6):1031-6.

76. Osmundson SS, Gould JB, Butwick AJ, Yeaton-Massey A, El-Sayed YY. Labor outcome at extremely advanced maternal age. Am J Obstet Gynecol. 2016; 214(3):362.e1-7.

77. Pallasmaa N, Ekblad U, Gissler M, Alanen A. The impact of maternal obesity, age, pre-eclampsia and insulin dependent diabetes on severe maternal morbidity by mode of delivery-a register-based cohort study. Arch Gynecol Obstet. 2015;291(2):311-8.

78. Platner MH, Ackerman C, Howland RE, Xu X, Pettker CM, Illuzzi JL, et al. Gestational weight gain and severe maternal morbidity at delivery hospitalization. Obstet Gynecol. 2019;133(3):515-24.

79. Ramage K, Grabowska K, Silversides C, Quan H, Metcalfe A. Association of adult congenital heart disease with pregnancy, maternal, and neonatal outcomes. JAMA Netw Open. 2019;2(5):e193667.

80. Ray JG, Park AL, Dzakpasu S, Dayan N, Deb-Rinker P, Luo W, et al. Prevalence of severe maternal morbidity and factors associated with maternal mortality in Ontario, Canada. JAMA Netw Open. 2018;1(7):e184571.

81. Reid LD, Creanga AA. Severe maternal morbidity and related hospital quality measures in Maryland. J Perinatol. 2018;38(8):997-1008.

82. Roberts CL, Ford JB, Algert CS, Bell JC, Simpson JM, Morris JM. Trends in adverse maternal outcomes during childbirth: a population-based study of severe maternal morbidity. BMC Pregnancy Childbirth. 2009;9:7.

83. Rosenbloom JI, Tuuli MG, Stout MJ, Young OM. Woolfolk, Macones GA, et al. a prediction model for severe maternal morbidity in laboring patients at term. Am J Perinatol. 2019;36(1):8-14.

84. Roy A, Peaceman A, Son M, Feinglass J. Maternal obstetric complication rates remain high in Illinois: a retrospective study, 2010-2015. Jt Comm J Qual Patient Saf. 2019;45(1):24-30.

85. Schummers L, Hutcheon JA, Hacker MR, VanderWeele TJ, Williams PL, McElrath TF, et al. Absolute risks of obstetric outcomes risks by maternal age at first birth: a population-based cohort. Epidemiology. 2018;29(3):37987.

86. Schummers L, Hutcheon JA, Bodnar LM, Lieberman E, Himes KP. Risk of adverse pregnancy outcomes by prepregnancy body mass index: a population-based study to inform prepregnancy weight loss counseling. Obstet Gynecol. 2015;125(1):133-43.

87. Shand AW, Chen JS, Selby W, Solomon M, Roberts CL. Inflammatory bowel disease in pregnancy: a population-based study of prevalence and pregnancy outcomes. BJOG. 2016;123(11):1862-70.

88. Siddiqui A, Azria E, Howell EA, Deneux-Tharaux C, Langer B, Dupont C, et al. Associations between maternal obesity and severe maternal morbidity: findings from the French EPIMOMS population-based study. Paediatr Perinat Epidemiol. 2019;33(1):7-16

89. Urquia ML, Wanigaratne S, Ray JG, Joseph KS. Severe maternal morbidity associated with maternal birthplace: a population-based register study. J Obstet Gynaecol Can. 2017;39(11):978-87.

90. Urquia ML, Glazier RH, Mortensen L, Nybo-Andersen AM, Small R, Davey MA, et al. Severe maternal morbidity associated with maternal birthplace in three high-immigration settings. Eur J Pub Health. 2015;25(4):620-5.

91. Vanderlaan J, Rochat R, Williams B, Dunlop A, Shapiro SE. Associations between hospital maternal service level and delivery outcomes. Womens Health Issues. 2019;29(3):252-8.

92. Wahlberg A, Roost M, Haglund B, Hogberg U, Essen B. Increased risk of severe maternal morbidity (near-miss) among immigrant women in Sweden: a population register-based study. BJOG. 2013;120(13):1605-11 discussion 1612

93. Wang ET, Ozimek JA, Greene N, Ramos L, Vyas N, Kilpatrick SJ, et al. Impact of fertility treatment on severe maternal morbidity. Fertil Steril. 2016;106(2): 423-6.

94. Young CB, Liu S, Muraca GM, Sabr Y, Pressey T, Liston RM, et al. Mode of delivery after a previous cesarean birth, and associated maternal and neonatal morbidity. CMAJ. 2018;190(18):e556-64.

95. Geller SE, Rosenberg D, Cox S, Brown M, Simonson L, Kilpatrick S. A scoring system identified near-miss maternal morbidity during pregnancy. J Clin Epidemiol. 2004;57:716-20.

96. Lain SJ, Hadfield RM, Raynes-Greenow CH, Ford JB, Mealing NM, Algert CS, et al. Quality of data in perinatal population health databases: a systematic review. Med Care. 2012;50(4):e7-e20.

97. Yasmeen S, Romano PS, Schembri ME, Keyzer JM, Gilbert WM. Accuracy of obstetric diagnoses and procedures in hospital discharge data. Am J Obstet Gynecol. 2006;194:992-1001.

98. American College of Obstetricians and Gynecologists (ACOG). Levels of maternal care. Obstetric Care Consensus No. 2. Obstet Gynecol. 2015;125(2): 502-15.

99. Agency for Healthcare Research and Quality. National Inpatient Sample Overview. Available from: https://www.hcup-us.ahrq.gov/nisoverview.jsp. Accessed 1 Sept 2020

100. Cauldwell M, Ghonim S, Uebing A, Swan L, Steer PJ, Gatzoulis M, et al. Preconception counseling, predicting risk and outcomes in women with mWHO 3 and 4 heart disease. Int J Cardiol. 2017;234:76-80.

101. California Maternal Quality Care Collaborative. OB Hemorrhage Toolkit v2.0. Available from: https://www.cmqcc.org/resources-tool-kits/toolkits/obhemorrhage-toolkit. Accessed 1 Sept 2020.

\section{Publisher's Note}

Springer Nature remains neutral with regard to jurisdictional claims in published maps and institutional affiliations.

\section{Ready to submit your research? Choose BMC and benefit from:}

- fast, convenient online submission

- thorough peer review by experienced researchers in your field

- rapid publication on acceptance

- support for research data, including large and complex data types

- gold Open Access which fosters wider collaboration and increased citations

- maximum visibility for your research: over $100 \mathrm{M}$ website views per year

At BMC, research is always in progress.

Learn more biomedcentral.com/submissions 